\title{
VALUATIVE ANALYSIS OF PLANAR PLURISUBHARMONIC FUNCTIONS
}

\author{
CHARLES FAVRE AND MATTIAS JONSSON
}

\begin{abstract}
We show that valuations on the ring $R$ of holomorphic germs in dimension 2 may be naturally evaluated on plurisubharmonic functions, giving rise to generalized Lelong numbers in the sense of Demailly. Any plurisubharmonic function thus defines a real-valued function on the set $\mathcal{V}$ of valuations on $R$ and-by way of a natural Laplace operator defined in terms of the tree structure on $\mathcal{V}$ - a positive measure on $\mathcal{V}$. This measure contains a great deal of information on the singularity at the origin. Under mild regularity assumptions, it yields an exact formula for the mixed Monge-Ampère mass of two plurisubharmonic functions. As a consequence, any generalized Lelong number can be interpreted as an average of valuations. Using our machinery we also show that the singularity of any positive closed $(1,1)$ current $T$ can be attenuated in the following sense: there exists a finite composition of blowups such that the pull-back of $T$ decomposes into two parts, the first associated to a divisor with normal crossing support, the second having small Lelong numbers.
\end{abstract}

\section{Contents}

Introduction

1. The valuative tree 4

2. Plurisubharmonic functions 9

3. Evaluating quasimonomial valuations on psh functions 12

4. Further properties 20

5. Potential theory on trees 22

6. Tree transforms of psh functions 26

7. Attenuation of singularities of currents 29

8. Intersection formula 33

$\begin{array}{ll}\text { References } & 36\end{array}$

Date: October 25, 2018.

1991 Mathematics Subject Classification. Primary: 32U25, Secondary: 13A18, 13H05.

Key words and phrases. Currents, Kiselman numbers, Laplace operator, Lelong numbers, plurisubharmonic functions, resolution of singularities, trees, valuations.

Second author supported by NSF Grant No DMS-0200614. 


\section{INTRODUCTION}

Valuation theory was a fundamental tool in the work of Zariski on desingularization of algebraic varieties. The key role in Zariski's approach was played by what is now called the Riemann-Zariski surface introduced in [Za]. It is by definition a collection of valuations. Our aim is to use a version of the Riemann-Zariski surface of the ring $R$ of holomorphic germs in dimension two to study local singularities of plurisubharmonic (psh) functions. Our thesis, as put forward in this paper and a forthcoming one [FJ2], is that valuations capture essentially all the information on a local singularity of a psh function in the plane.

Our study is local. We say that a psh function $u$, defined near the origin in $\mathbf{C}^{2}$, has a singularity (at the origin) when $u(0)=-\infty$. To study such a singularity, several quantities have been introduced. The limit of $(\log r)^{-1} \sup \{u(q) ;|q| \leq r\}$ as $r \rightarrow 0$ exists [Le] and is called the Lelong number of $u$. This number gives information on the growth of $u$ around its singular point. It coincides with the multiplicity of the curve $\{\psi=0\}$ when $u=\log |\psi|, \psi \in R$.

More generally, given local coordinates $(x, y)$ and weights $a, b>0$ one can [Ki1] associate to $u$ the limit of $(a b / \log r) \sup \left\{u(x, y) ;|x| \leq r^{1 / a},|y| \leq r^{1 / b}\right\}$ as $r \rightarrow 0$. This limit is known as the Kiselman number and gives more precise information on the singularity. The Kiselman number of $u=\log |\psi|, \psi \in R$ coincides with $\nu(\psi)$ where $\nu$ denotes the monomial valuation sending $x$ to $a$ and $y$ to $b$.

Demailly [De2] put these constructions into a more general framework. He defined a notion of psh weight (see Section 2.5 below) and attached to any psh function $u$ and any weight $\varphi$ a generalized Lelong number $\nu_{\varphi}(u) \geq 0$.

Three questions naturally arise: 1) Are Demailly's generalized Lelong numbers related to valuations? 2) When do two psh weights define the same generalized Lelong number? 3) What information on the singularity of $u$ can be recovered from the generalized Lelong numbers?

In this paper and in [FJ2] we address these three questions. As an answer to the first, we prove that any valuation may be evaluated on psh functions (Theorem 3.1), and as such defines a generalized Lelong number (Proposition 3.9). Conversely we prove that any generalized Lelong number associated to a (sufficiently regular) weight is an average of valuations (Theorem 8.7), giving an answer to the second question. The averaging is with respect to a positive measure, the tree measure of the weight.

The tree measure $\rho_{u}$ of a psh function $u$ is a positive measure (of mass equal to the Lelong number of $u$ ) on a space $\mathcal{V}$ of valuations defined below. It is computed in terms of valuations, hence is determined by the collection of all generalized Lelong numbers of $u$. A slightly vague answer to the third question above is therefore that the tree measure $\rho_{u}$ contains essentially complete information on the singularity of $u$. Let us outline four ways to make this assertion more precise.

First, two psh functions have the same tree measure iff their pullbacks by any composition of point blowups above the origin have the same Lelong numbers at any point on the exceptional divisor. 
Second, we show in [FJ2] that the tree measure $\rho_{u}$ determine the multiplier ideals of all multiples of a psh function $u$. In that paper we also use valuations to give an affirmative answer to the "openness conjecture" by Demailly and Kollàr.

Third, we use tree measures to prove that every positive closed $(1,1)$ current $T$, defined near the origin, admits an attenuation of singularities: there exists a finite composition of blowups, such that the pullback of $T$ decomposes into two parts, the first associated to a divisor with normal crossing support, and the second having arbitrarily small Lelong numbers everywhere. The corresponding global result - for currents on compact complex surfaces - is an easy consequence, and has recently been proved by V. Guedj $[G]$ in a more elementary way. His proof follows the method by Mimouni [Mi], who was the first, to our knowledge, to study singularities of psh functions through sequences of blowups. However, the global result does not imply the local one, and in fact our method gives stronger control on the second part of the decomposition, even in the global case.

Fourth, we explore the relationship between the singularities of two individual psh functions $u$ and $v$, and the mass at the origin of the mixed Monge-Ampère measure $d d^{c} u \wedge d d^{c} v$. For general $u$ and $v$, only inequalities in terms of Lelong or Kiselman numbers were known, see e.g. [De1, Chapter 3], [Ra]. Here we obtain much sharper estimate for $d d^{c} u \wedge d d^{c} v\{0\}$ in terms of the tree measures $\rho_{u}$ and $\rho_{v}$. In fact, we obtain an exact formula under a mild regularity assumption on either $u$ or $v$. The fact that any generalized Lelong number of a (Hölder) psh weight is an average of valuations is a consequence of this formula. The corresponding statement for homogeneous psh functions was obtained by Rashkovskii.

Let us describe more precisely the content of the article. The Riemann-Zariski surface is classically endowed with a non-Hausdorff topology. We described in [FJ1] a natural way to turn it into a Hausdorff compact space, called the valuative tree, that is well suited for analysis. This space is the set $\mathcal{V}$ of all normalized $\mathbf{R}_{+} \cup\{\infty\}$ valued valuations on $R$ centered at the maximal ideal. The structure of $\mathcal{V}$, essential to our analysis here, is described in great detail in [FJ1]; we recall in Section 1 the results that we use in the present paper. Of particular importance is the fact that $\mathcal{V}$ has a natural tree structure: it is made up of pieces (segments) that are canonically parameterized by real intervals. The main difficulty in generalizing our results to higher dimensions lies in the fact that the structure of the Riemann-Zariski variety is not well understood in dimension three or higher.

Section 2 contains basic facts on psh functions. In particular we recall the powerful approximation technique due to Demailly. We also review some facts on Kiselman numbers and (generalized) Lelong numbers.

In Section 3 we define $\nu(u)$ for a (quasimonomial) valuation $\nu \in \mathcal{V}$ and a psh function $u$. The function $u \mapsto \nu(u)$ may be roughly characterized as the minimal, upper semicontinuous function satisfying $\nu(\log |\psi|)=\nu(\psi)$ for all holomorphic germs $\psi$. Our approach is as follows. If $\nu$ is monomial, then we define $\nu(u)$ as a Kiselman number. We extend this to $\nu$ in the dense subtree $\mathcal{V}_{\mathrm{qm}}$ of $\mathcal{V}$ consisting of quasimonomial valuations. Such valuations can be made monomial by a birational morphism, a fact which allows us to define $\nu(u)$ as a growth rate of $u$ in a semianalytic characteristic region associated to $\nu$. We also identify $\nu(u)$ as a generalized Lelong number. 
In Section 4 we further investigate the function $u \mapsto \nu(u)$. In particular we show that a divisorial valuation may be interpreted as the (normalized) Lelong number at a generic point on a suitable exceptional component.

For a fixed psh function $u$ we call the function $\nu \mapsto \nu(u)$ on $\mathcal{V}_{\mathrm{qm}}$ the tree transform of $u$. It has very strong concavity properties (some of which were noticed by Kiselman [Ki1]) with respect to the tree structure on $\mathcal{V}$. In Section 5, we describe a class of functions $g: \mathcal{V}_{\mathrm{qm}} \rightarrow \mathbf{R}_{+}$called tree potentials and having exactly these concavity properties. These functions were introduced and studied extensively in [FJ1]. The set $\mathcal{P}$ of tree potentials is the smallest closed convex cone in $\mathcal{V}_{\mathrm{qm}}^{\mathbf{R}}$, containing all functions on $\mathcal{V}_{\mathrm{qm}}$ of the form $\nu \mapsto \nu(\phi)$ for $\phi \in R$, and closed under minima. Theorem 5.9 asserts that there is a one-to-one correspondence between $\mathcal{P}$ and the set $\mathcal{M}$ of positive measures on $\mathcal{V}$. The map $\mathcal{M} \rightarrow \mathcal{P}$ is defined using a natural intersection product on $\mathcal{V}$. Its inverse is a natural Laplace operator $\Delta: \mathcal{P} \rightarrow \mathcal{M}$.

In Section 6 we prove that the tree transform of any psh function $u$ defines a tree potential $g_{u}$, hence a positive measure $\rho_{u}=\Delta g_{u}$ on $\mathcal{V}$, called the tree measure of $u$. As noted above, the tree measure gives an extremely fine description of the singularity. Although a complete characterization of measures arising from psh functions seems hard to obtain, we provide some partial results.

Attenuation of singularities for currents, as described above, is proved in Section 7. Our proof is constructive in the sense that the composition of blowups may be recovered from the tree measure of the current. Basically, the idea is to partition the valuative tree into subsets, each of which has small mass for the tree measure. The subsets define points on the exceptional divisor and the mass gives a bound for the Lelong number at the point. In our approach, the finiteness of a suitable intersection product, as used in the analysis by Mimouni and Guedj, is replaced by the finiteness of the tree measure.

In Section 8, we give sharp estimates of the mixed Monge-Ampère measure $d d^{c} u \wedge d d^{c} v\{0\}$ in terms of the tree measures of $u$ and $v$. We also prove that generalized Lelong numbers are averages of valuations, see Theorem 8.7. All results in this section rely on a reduction to the algebraic case, using Demailly's approximation technique.

\section{The Valuative tree}

In this section we give a brief review of the valuative tree as described in [FJ1]. Throughout the paper, we set $R=\mathcal{O}_{0}$, the ring of holomorphic germs at the origin in $\mathbf{C}^{2}$. This is a local ring. Its (unique) maximal ideal $\mathfrak{m}$ is the set of germs vanishing at the origin, and its residue field is $\mathbf{C}$. We write $(\hat{R}, \hat{\mathfrak{m}})$ for the completion of $(R, \mathfrak{m})$. It is the ring of formal power series in two complex variables.

1.1. Valuations. We consider the space $\mathcal{V}$ of centered, normalized valuations on $R$, i.e. the set of functions $\nu: R \rightarrow[0, \infty]$ satisfying:

(i) $\nu\left(\psi \psi^{\prime}\right)=\nu(\psi)+\nu\left(\psi^{\prime}\right)$ for all $\psi, \psi^{\prime}$;

(ii) $\nu\left(\psi+\psi^{\prime}\right) \geq \min \left\{\nu(\psi), \nu\left(\psi^{\prime}\right)\right\}$ for all $\psi, \psi^{\prime}$;

(iii) $\nu(0)=\infty,\left.\nu\right|_{\mathbf{C}^{*}}=0, \nu(\mathfrak{m}):=\min \{\nu(\psi) \mid \psi \in \mathfrak{m}\}=1$. 
Then $\mathcal{V}$ is equipped with a natural partial ordering: $\nu \leq \mu$ iff $\nu(\psi) \leq \mu(\psi)$ for all $\psi \in \mathfrak{m}$. The multiplicity valuation $\nu_{\mathfrak{m}}$ defined by $\nu_{\mathfrak{m}}(\psi)=m(\psi)=\max \left\{k \mid \psi \in \mathfrak{m}^{k}\right\}$ is the unique minimal element of $\mathcal{V}$.

Note that any valuation on $R$ extends uniquely to a valuation on its completion $\hat{R}$, hence the valuation spaces attached to $R$ and $\hat{R}$ are isomorphic.

1.2. Curve valuations. Some natural maximal elements are the curve valuations defined as follows. To each irreducible (possibly formal) curve $C$ we associate $\nu_{C} \in \mathcal{V}$ defined by $\nu_{C}(\psi)=C \cdot\{\psi=0\} / m(C)$, where "." denotes intersection multiplicity. If $C$ is defined by $\phi \in \hat{\mathfrak{m}}$, then we also write $\nu_{C}=\nu_{\phi}$.

The set $\mathcal{C}$ of local irreducible curves carries a natural (ultra)metric in which $\mathcal{C}$ has diameter 1. It is given by $d_{\mathcal{C}}(C, D)=m(C) m(D) / C \cdot D$.

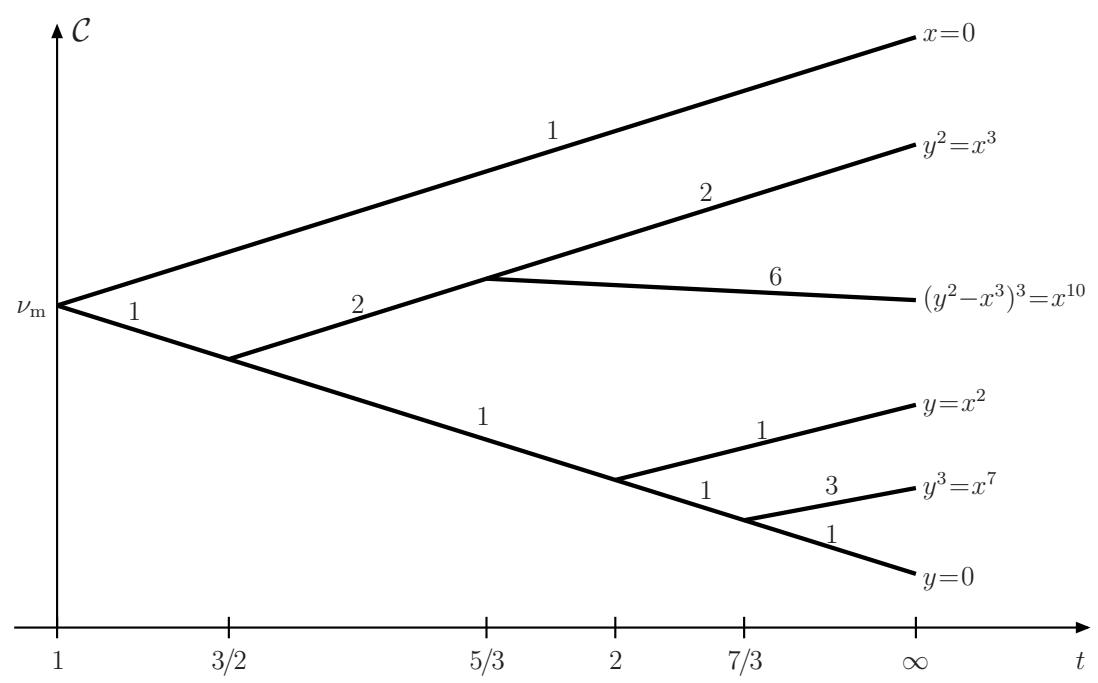

Figure 1. The valuative tree. The segments consist of valuations of the form $\nu_{\phi, t}$, where $\phi=x, y^{2}-x^{3}, \ldots, y$ and the skewness parameter $t$ ranges from 1 to $\infty$. Skewness $t=1$ gives the multiplicity valuation $\nu_{\mathfrak{m}}$ and skewness $t=\infty$ the curve valuation $\nu_{\phi}$. The integer label above a segment indicates multiplicity (Section 1.6).

1.3. Quasimonomial valuations. Arguably the most important valuations in $\mathcal{V}$ are the quasimonomial ones. ${ }^{1}$ They are of the form $\nu_{C, t}$, where $C \in \mathcal{C}$ and $t \in[1, \infty)$, and satisfy $\nu_{C, t}(\psi)=\min \left\{\nu_{D}(\psi) \mid d_{\mathcal{C}}(C, D) \leq t^{-1}\right\}$. We have $\nu_{C, s}=$ $\nu_{D, t}$ iff $s=t \geq d_{\mathcal{C}}(C, D)^{-1}$ (see [FJ1, Theorem 3.57]). Thus $\mathcal{V}_{\mathrm{qm}}$, the set of all quasimonomial valuations, is naturally a quotient of $\mathcal{C} \times[1, \infty)$, and has a natural tree structure: if $\nu, \nu^{\prime} \in \mathcal{V}_{\mathrm{qm}}$ and $\nu<\nu^{\prime}$, then the segment $\left[\nu, \nu^{\prime}\right]=\left\{\mu \in \mathcal{V}_{\mathrm{qm}} \mid \nu \leq\right.$ $\left.\mu \leq \nu^{\prime}\right\}$ is isomorphic to a compact real interval, see Figure 1. We set $\nu_{\phi, t}:=\nu_{C, t}$

\footnotetext{
${ }^{1}$ A quasimonomial valuation can be made monomial (i.e. completely determined by its values on a pair of local coordinates $(x, y)$ ) by a birational morphism: see Section 3.3. Quasimonomial valuations are also known as Abhyankar valuations of rank 1.
} 
when $C=\{\phi=0\}$. The value $\nu_{C, t}(\psi)$ can be interpreted as the order of vanishing of $\psi$ in a suitable "cone" around the curve $C$ of size depending on $t$. We refer to Section 3.1 and Proposition 3.6 for more precise statements. Figure 2 depicts such a cone. Quasimonomial valuations are of two types: divisorial and irrational,

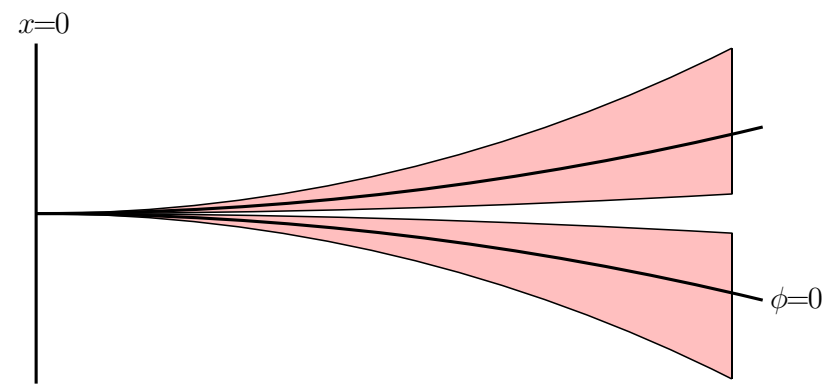

Figure 2. A characteristic region in $\mathbf{C}^{2}$.

depending on whether the parameter $t$ is rational or irrational. ${ }^{2}$ The full space $\mathcal{V}$ is the completion of $\mathcal{V}_{\mathrm{qm}}$ in the sense that every element in $\mathcal{V}$ is the limit of an increasing sequence in $\mathcal{V}_{\mathrm{qm}}$. It is hence also naturally a tree, called the valuative tree. The ends of $\mathcal{V}$ are exactly the elements of $\mathcal{V} \backslash \mathcal{V}_{\mathrm{qm}}$ and are either curve valuations or infinitely singular valuations. ${ }^{3}$

1.4. Skewness and intersection multiplicity. An important invariant of a valuation is its skewness $\alpha$ defined by $\alpha(\nu)=\sup \{\nu(\phi) / m(\phi) \mid \phi \in \mathfrak{m}\}$. Skewness naturally parameterizes the trees $\mathcal{V}_{\mathrm{qm}}$ and $\mathcal{V}$ in the sense that $\alpha: \mathcal{V}_{\mathrm{qm}} \rightarrow[0, \infty)$ is strictly increasing and restricts to a bijection onto its image on any segment; indeed $\alpha\left(\nu_{\phi, t}\right)=t$ for any $\nu_{\phi, t} \in \mathcal{V}_{\mathrm{qm}}$. See [FJ1, Section 3.3]. Thus divisorial (irrational) valuations have rational (irrational) skewness. Curve valuations have infinite skewness whereas the skewness of an infinitely singular valuations may or may not be finite.

The tree structure on $\mathcal{V}$ implies that any collection $\left(\nu_{i}\right)_{i \in I}$ of valuations in $\mathcal{V}$ admits an infimum $\wedge_{i} \nu_{i}$. Together with skewness, this allows us to define an intersection product on $\mathcal{V}$ : we set $\nu \cdot \mu:=\alpha(\nu \wedge \mu) \in[1, \infty]$. This is a normalized extension of the intersection product on $\mathcal{C}$ as $C \cdot D=\left(\nu_{C} \cdot \nu_{D}\right) m(C) m(D)$. If $\nu \in \mathcal{V}$ and $\phi \in \mathfrak{m}$ is irreducible, then $\nu(\phi)=m(\phi)\left(\nu \cdot \nu_{\phi}\right)$. Moreover, if $\nu(\phi)$ is irrational, then $\nu=\nu_{\phi, t}$ with $t=\nu(\phi) / m(\phi)$.

1.5. Tangent space and weak topology. Let $\mu$ be a valuation in $\mathcal{V}$. Declare $\nu, \nu^{\prime} \in \mathcal{V} \backslash\{\mu\}$ to be equivalent if the segments $\left.] \mu, \nu\right]$ and $\left.] \mu, \nu^{\prime}\right]$ intersect. An equivalence class is called a tangent vector at $\mu$ and the set of tangent vectors at $\mu$, the tangent space, denoted by $T \mu$. If $\vec{v}$ is a tangent vector, we denote by $U(\vec{v})$ the set of points in $\mathcal{V}$ defining the equivalence class $\vec{v}$. The points in $U(\vec{v})$ are said to represent $\vec{v}$.

\footnotetext{
${ }^{2} \mathrm{~A}$ quasimonomial valuation $\nu$ is irrational iff $\nu(R) \not \subset \mathbf{Q}$, hence the name.

${ }^{3}$ The latter are represented by infinite Puiseux series whose exponents are rational numbers with unbounded denominators. See Section 1.9.
} 
A point $\mu$ in the tree $\mathcal{V}$ is an end, a regular point, or a branch point when $T \mu$ contains one, two, or three or more points, respectively. In terms of valuations: the ends of $\mathcal{V}$ are curve and infinitely singular valuations; the regular points irrational valuations; and the branch points divisorial valuations, at which the tangent space is in bijection with the complex projective line $\mathbf{P}^{1}$ and hence uncountable. See [FJ1, Proposition 3.20].

We endow $\mathcal{V}$ with the weak topology, generated by the sets $U(\vec{v})$ over all tangent vectors $\vec{v}$; this turns $\mathcal{V}$ into a compact (Hausdorff) space. The weak topology on $\mathcal{V}$ is characterized by $\nu_{k} \rightarrow \nu$ iff $\nu_{k}(\phi) \rightarrow \nu(\phi)$ for all $\phi \in R$; see [FJ1, Theorem 5.1].

1.6. Multiplicities. (See [FJ1, Section 3.4]) By setting $m(\nu)=\min \{m(C) \mid C \in$ $\left.\mathcal{C}, \nu_{C} \geq \nu\right\}$ we extend the notion of multiplicity from $\mathcal{C}$ to $\mathcal{V}_{\mathrm{qm}}$. Clearly $m$ : $\mathcal{V}_{\mathrm{qm}} \rightarrow \mathbf{N}$ is increasing and hence extends to all of $\mathcal{V}$. In fact $m(\nu)$ divides $m(\mu)$ whenever $\nu \leq \mu$. The infinitely singular valuations are characterized as having infinite multiplicity.

As $m$ is increasing and integer valued, it is piecewise constant on any segment $\left[\nu_{\mathfrak{m}}, \nu_{\phi}\right]$, where $\phi \in \mathcal{C}$. This implies that $m(\vec{v})$ is naturally defined for any tangent vector $\vec{v}$. If $\nu$ is nondivisorial, then $m(\vec{v})=m(\nu)$ for any $\vec{v} \in T \nu$.

If $\nu$ is divisorial, then the situation is more complicated. We refer to [FJ1, Proposition 3.39] for a precise discussion. Suffice it to say that there exists an integer $b(\nu)$, divisible by $m(\nu)$, such that $m(\vec{v})=b(\nu)$ for all but at most two tangent vectors $\vec{v}$ at $\nu$. We call $b(\nu)$ the generic multiplicity of $\nu$.

1.7. Approximating sequences. (See [FJ1, Section 3.5]). Consider a quasimonomial valuation $\nu \in \mathcal{V}_{\mathrm{qm}}$. The multiplicity $m$ is integer-valued and piecewise constant on the segment $\left[\nu_{\mathfrak{m}}, \nu\right]$ and therefore has a finite number $g$ (possibly zero) of jumps. Thus there are divisorial valuations $\nu_{i}, 0 \leq i \leq g$ and integers $m_{i}$, such that

$$
\nu_{\mathfrak{m}}=\nu_{0}<\nu_{1}<\cdots<\nu_{g}<\nu_{g+1}=\nu
$$

and $m(\mu)=m_{i}$ for $\left.\left.\mu \in\right] \nu_{i}, \nu_{i+1}\right], 0 \leq i \leq g$. We call the sequence $\left(\nu_{i}\right)_{i=0}^{g}$ the approximating sequence associated to $\nu$. It also plays a prominent role in [Sp].

The concept of approximating sequences extends naturally to valuations that are not quasimonomial: for curve valuations the sequences are still finite, for infinitely singular valuations they are infinite.

1.8. Thinness. (See [FJ1, Section 3.6]). Skewness $\alpha$ is a parameterization of $\mathcal{V}$ that does not "see" multiplicities. Another parameterization, of crucial importance, is thinness $A$, defined as follows. If $\nu \in \mathcal{V}_{\text {qm }}$ then

$$
A(\nu)=2+\int_{\nu_{\mathfrak{m}}}^{\nu} m(\mu) d \alpha(\mu)
$$

In terms of (1.1) we have $A(\nu)=2+\sum_{0}^{g} m_{i}\left(\alpha_{i+1}-\alpha_{i}\right)$ with $\alpha_{i}=\alpha\left(\nu_{i}\right)$. Note that $A(\nu) \leq 1+m(\nu) \alpha(\nu)$. Just like skewness, we may define $A(\nu)$ also for $\nu \notin \mathcal{V}_{\mathrm{qm}}$. 
1.9. Puiseux expansions. (See [FJ1, Chapter 4]). Valuations in $\mathcal{V}$ can be encoded (nonuniquely) by Puiseux series. We describe this here in the case of an end $\nu \in \mathcal{V}$, i.e. an infinitely singular or curve valuation. Pick local coordinates $(x, y)$ such that $\nu_{\mathfrak{m}}<\nu \wedge \nu_{y}$. Then $\nu$ is represented by a series $\hat{\phi}=\sum_{i \geq 1} a_{i} x^{\hat{\beta}_{i}}$, where $a_{i} \in \mathbf{C}^{*}$ and $\hat{\beta}_{i}>1$ form an increasing sequence of rational numbers. For $\psi \in R$, $\psi(t, \hat{\phi}(t))=\sum b_{j} t^{\hat{\gamma}_{j}}$ is a Puiseux series and $\nu(\psi)=\min \left\{\hat{\gamma}_{j} ; b_{j} \neq 0\right\}$. Moreover, $A(\nu)=1+\lim _{i \rightarrow \infty} \hat{\beta}_{i}$ if the Puiseux series is infinite and $A(\nu)=\infty$ otherwise.

If the denominators of the $\hat{\beta}_{i}$ 's are bounded, then $\nu$ is a curve valuation. Otherwise, $\nu$ is infinitely singular. In the latter case we can define $C_{n} \in \mathcal{C}$ to be the irreducible curve associated to the truncated Puiseux expansion $\hat{\phi}_{n}=\sum_{1}^{n} a_{i} x^{\hat{\beta}_{i}}$. Then $\nu_{C_{n}}$ converges weakly to $\nu$.

1.10. Geometric interpretation of divisorial valuations. (See [FJ1, Chapter 6]). All divisorial valuations $\nu$ arise as follows: there exists a (proper) modification $\pi: X \rightarrow\left(\mathbf{C}^{2}, 0\right)$-in our case a finite composition of point blowups - and an exceptional component $E$ (i.e. $E$ is an irreducible component of the exceptional divisor $\left.\pi^{-1}(0)\right)$ such that $\nu=: \nu_{E}$ is equivalent to $\pi_{*} \operatorname{div}_{E}$, where $\operatorname{div}_{E}$ denotes the order of vanishing along $E$. More precisely:

- $\nu=b^{-1} \pi_{*} \operatorname{div}_{E}$, where $b=b(\nu)$ is the generic multiplicity at $\nu$;

- $A(\nu)=a / b$, where $a-1$ is equal to the order of vanishing along $E$ of the Jacobian determinant of $\pi$.

In fact, the pair $(a, b)$, which is called the Farey weight of $E$ in [FJ1], can be obtained in a purely combinatorial way (see also $[\mathrm{HP}]$ ). This combinatorial procedure can be used to recover the full tree structure (partial ordering, thinness and multiplicity) on the valuative tree $\mathcal{V}$, and the assertions above are consequences of a much more precise result: see [FJ1, Theorem 6.22].

The generic multiplicity $b\left(\nu_{E}\right)$ is the multiplicity of any curvette for $\nu_{E}$, i.e. any irreducible curve $C$ whose strict transform under $\pi$ is smooth and intersects $E$ transversely at a smooth point on $E$. For any curvette we have $\nu_{C}>\nu_{E}$. See [FJ1, Section 6.6.1].

In Section 7, we shall need several results relating divisorial valuations and their location inside $\mathcal{V}$ to dual graphs of modifications. See Figure 3.

Proposition 1.1. [FJ1, Proposition 6.37] Let $\pi: X \rightarrow\left(\mathbf{C}^{2}, 0\right)$ be a modification. Pick a point $p \in \pi^{-1}(0)$, and let $\nu_{p}$ be the divisorial valuation associated to the blowup at $p$.

(i) If $p$ belongs to a unique exceptional component $E$ of $\pi$, then $\nu_{p}>\nu_{E}$; $\nu_{p}$ does not represent the same tangent vector at $\nu_{E}$ as $\nu_{E^{\prime}}$ for any other exceptional component $E^{\prime}$; and the multiplicity is constant equal to $b\left(\nu_{p}\right)=$ $b\left(\nu_{E}\right)$ on the segment $\left.] \nu_{E}, \nu_{p}\right]$.

(ii) If $p$ is the intersection of two exceptional components $E$ and $E^{\prime}$, then $\nu_{E}<$ $\nu_{p}<\nu_{E^{\prime}}\left(\right.$ or $\left.\nu_{E^{\prime}}<\nu_{p}<\nu_{E}\right)$; and $b\left(\nu_{p}\right)=b\left(\nu_{E}\right)+b\left(\nu_{E^{\prime}}\right)$.

We shall also use the following direct consequence of [FJ1, Corollary 6.39]. 


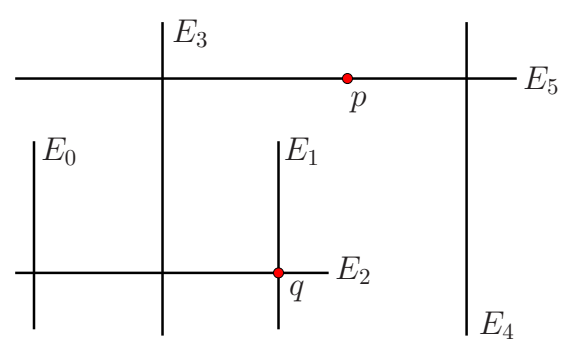

$X$

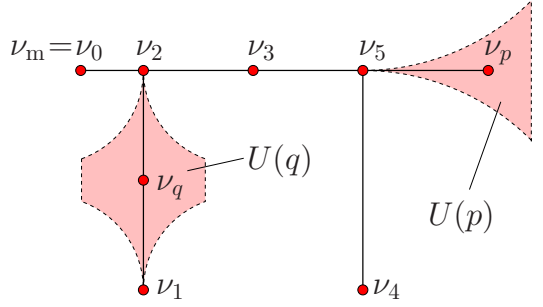

V

Figure 3. Dual graphs and valuations. To the left is the total space of a composition $\pi: X \rightarrow\left(\mathbf{C}^{2}, 0\right)$ of six point blowups. To the right is the dual graph of $\pi$ embedded in the valuative tree $\mathcal{V}$. To the exceptional component $E_{i}$ is associated the valuation $\nu_{i}$. The valuations $\nu_{p}$ and $\nu_{q}$, obtained by blowing up the points $p$ and $q$, illustrate case (i) and (ii) of Proposition 1.1, respectively. The open subsets $U(p)$ and $U(q)$ of $\mathcal{V}$ consist of all valuations in $\mathcal{V}$ whose center on $X$ is $p$ and $q$, respectively.

Lemma 1.2. Let $\pi: X \rightarrow\left(\mathbf{C}^{2}, 0\right)$ be a modification, and let $E, E^{\prime}$ be two exceptional components that intersect in $X$. Then $\nu_{E}, \nu_{E^{\prime}}$ are comparable and

$$
\left|\alpha\left(\nu_{E^{\prime}}\right)-\alpha\left(\nu_{E}\right)\right|=\frac{1}{b\left(\nu_{E^{\prime}}\right) b\left(\nu_{E}\right)} .
$$

\section{Plurisubharmonic Functions}

In this section we recall some facts on plurisubharmonic (psh) functions that we will need. General references are [De1] and [Hö].

2.1. Basics. Unless otherwise specified, all psh functions are defined in some neighborhood of the origin in $\mathbf{C}^{2}$. We write $d d^{c} u=\frac{i}{2 \pi} \partial \bar{\partial} u$.

If $\psi$ is a holomorphic germ, i.e. $\psi \in R$, then $\log |\psi|$ is psh. More generally, if $\psi_{i} \in R, 1 \leq i \leq n$, then $u=\log \left(\sum_{1}^{n}\left|\psi_{i}\right|^{2}\right)$ is psh. A psh function $u$ such that $u-c \log \left(\sum_{1}^{n}\left|\psi_{i}\right|^{2}\right)$ is locally bounded for some $\psi_{i} \in R$ and $c>0$, is said to have logarithmic singularities.

We shall use the following consequence of Harnack's inequality:

Lemma 2.1. If $u \leq 0$ is psh in the bidisk $|x|<r_{1},|y|<r_{2}$, then

$$
\sup _{|x| \leq \varepsilon \rho_{1},|y| \leq \varepsilon \rho_{2}} u \leq \frac{\left(1-\varepsilon^{2}\right)^{2}}{(1+\varepsilon)^{4}} \iint u\left(\rho_{1} e^{i \theta_{1}}, \rho_{2} e^{i \theta_{2}}\right) \frac{d \theta_{1} d \theta_{2}}{(2 \pi)^{2}},
$$

whenever $\rho_{i} \in\left(0, r_{i}\right), i=1,2$ and $\varepsilon \in(0,1)$.

2.2. Demailly approximation. Our study uses in a crucial way a powerful technique, developed by Demailly, of approximating general psh functions by psh functions with logarithmic singularities. Let us summarize the main ingredients. See Theorem 4.2 and its proof in [DK] for details. 
Let $u$ be a psh function on a fixed ball $B$ containing the origin. For any real number $n>0$, we let $\mathcal{H}_{n u}(B)$ be the Hilbert space of holomorphic functions $f$ on $B$ such that $\|f\|_{n u}:=\int_{B}|f|^{2} \exp (-2 n u) d V<+\infty$, and define

$$
u_{n}=\frac{1}{2 n} \log \sum_{1}^{\infty}\left|g_{n k}\right|^{2}=\frac{1}{n} \log \sup \left\{|f| ;\|f\|_{n u} \leq 1\right\},
$$

where $g_{n k}$ is an orthonormal basis of $\mathcal{H}_{n u}(B)$. Then $u_{n}$ converges to $u$ in a rather strong sense: there exists $C>0$ such that for any $r>0$ small enough

$$
u(p)-\frac{C}{n} \leq u_{n}(p) \leq \sup _{B(p, r)} u+\frac{C}{n}-\frac{2}{n} \log r .
$$

In particular this implies $u_{n} \rightarrow u$ in $L_{\mathrm{loc}}^{1}$. It is the left hand inequality of (2.1) that is the harder one to establish - it is a consequence of the Ohsawa-Takegoshi extension theorem.

It is also convenient to truncate the infinite sum defining $u_{n}$. More precisely, given $n$ and a smaller ball $B^{\prime} \Subset B$ containing the origin, there exists $k_{0}=$ $k_{0}\left(u, n, B^{\prime}\right)<\infty$ such that $\sum_{1}^{\infty}\left|g_{n k}\right|^{2} \leq C \sum_{1}^{k_{0}}\left|g_{n k}\right|^{2}$ on $B^{\prime}$, for some $C>0$. Thus $u_{n}-(2 n)^{-1} \log \sum_{1}^{k_{0}}\left|g_{n k}\right|^{2}$ is bounded, so $u_{n}$ has logarithmic singularities.

In Section 8 we will work with the class of psh functions $u$ for which $e^{u}$ is Hölder continuous. This class, which appears at several points in the work of Demailly (see e.g. [DK, Theorem 2.5]), contains all psh functions with logarithmic singularities, but not, for instance, the function $u(p)=-\sqrt{-\log \|p\|}$.

One consequence of Hölder continuity is that the right hand side of (2.1) can be improved. Assume $u(0)=-\infty$ and that $e^{u}$ is Hölder continuous. Then we may find $c>0$ so that $\left|e^{u(p)}-e^{u\left(p^{\prime}\right)}\right| \leq\left\|p-p^{\prime}\right\|^{c}$ for $p, p^{\prime}$ in a small ball around the origin. Thus $\sup _{B(p, r)} u \leq \log \left(e^{u(p)}+r^{c}\right)$ for any $p$ and $r>0$ small enough. When $u(p)=-\infty,(2.1)$ yields $u_{n}(p)=-\infty$ for $n>2 / c$ by letting $r \rightarrow 0$. If $u(p)>-\infty$, then choosing $r=e^{u(p) / c}$ we infer from (2.1) the estimate

$$
u-\frac{C}{n} \leq u_{n} \leq\left(1-\frac{2}{n c}\right) u+\frac{C}{n},
$$

on some neighborhood $B^{\prime}$ of the origin and some constant $C>0$ (both independent of $n$ ).

2.3. Kiselman numbers. As we will see, the evaluation of a quasimonomial valuation on psh functions can be viewed as the pushforward under a birational morphism of a Kiselman number, so we recall some properties of the latter. The original references are [Ki1, Ki2]; see also [De1, Chapter III, Example 6.11].

Consider a psh function $u$, fix local coordinates $(x, y)$ and real numbers $a, b>0$. The Kiselman number of $u$ with weights $(a, b)$ is defined as

$$
\nu_{a, b}^{x, y}(u):=\lim _{r \rightarrow 0} \frac{a b}{\log r} \sup \left\{u ;|x|<r^{\frac{1}{a}},|y|<r^{\frac{1}{b}}\right\} .
$$

This limit exists. By the maximum principle we could equivalently take the supremum over either the torus $\left\{|x|=r^{1 / a},|y|=r^{1 / b}\right\}$ or over the open set 
$\left\{|x|<r^{1 / a},|y|<|x|^{a / b}\right\}$. Another definition of the Kiselman number, the equivalence of which to the previous definitions follows from Lemma 2.1, is given by

$$
\nu_{a, b}^{x, y}(u)=\lim _{r \rightarrow 0} \frac{a b}{\log r} \iint u\left(r^{\frac{1}{a}} e^{i \theta}, r^{\frac{1}{b}} e^{i \vartheta}\right) \frac{d \theta d \vartheta}{(2 \pi)^{2}} .
$$

In both regions $\left\{|x|<r^{1 / a},|y|<r^{1 / b}\right\}$ and $\left\{|x|<r^{1 / a},|y|<|x|^{a / b}\right\}$ we have

$$
u(p) \leq \frac{1}{\min \{a, b\}} \nu_{a, b}^{x, y}(u) \log \|p\|+O(1) \quad \text { as } p \rightarrow 0 .
$$

In the particular case $a=b=1$, the Kiselman number reduces to the Lelong number of $u$, originally defined in [Le]. We denote it by $\nu^{\mathrm{L}}(u)$. The Lelong number of the positive closed current $T=d d^{c} u$ can be also computed as follows. Define $\omega=d d^{c}\left(|x|^{2}+|y|^{2}\right)$, and let $\|T\|:=T \wedge \omega$ be the trace measure of $T$. Then

$$
\nu^{\mathrm{L}}(T)=\lim _{r \rightarrow 0} \frac{1}{\pi r^{2}}\|T\|[B(0, r)],
$$

where $B(0, r)$ denotes the ball of center 0 and radius $r$.

2.4. Intersection of currents. Let us recall a few facts about intersection of currents. See [Du, Section 4.1] for more details. Pick two psh functions $u, v$. When $u$ is locally integrable with respect to the trace measure of $d d^{c} v$, we say that the wedge product $d d^{c} u \wedge d d^{c} v$ is admissible, and we define $d d^{c} u \wedge d d^{c} v=d d^{c}\left(u d d^{c} v\right)$. In dimension two, it is a fact proved by Sibony, see [Du], that if $\left(u_{n}\right)_{1}^{\infty}$ and $\left(v_{n}\right)_{1}^{\infty}$ are sequences of psh functions such that $u_{n} \geq u, v_{n} \geq v, u_{n} \rightarrow u$ and $v_{n} \rightarrow v$ in $L_{\text {loc }}^{1}$, then $d d^{c} u_{n} \wedge d d^{c} v_{n}$ is admissible for all $n$ and

$$
d d^{c} u_{n} \wedge d d^{c} v_{n} \longrightarrow d d^{c} u \wedge d d^{c} v
$$

weakly, as $n \rightarrow \infty$. As follows from (2.1), we may apply (2.7) when $u_{n}$ and $v_{n}$ are Demailly approximants of $u$ and $v$, respectively.

Equation (2.7) may also be used to show that the condition of $d d^{c} u \wedge d d^{c} v$ being admissible is symmetric in $u$ and $v$.

2.5. Generalized Lelong numbers. We now introduce some terminology and definitions taken from [De1, Chapter III] (see also the original article [De2]). A psh function $\varphi$ such that $\varphi^{-1}\{-\infty\}=\{0\}$, and $e^{\varphi}$ is continuous is called a psh weight. If $\varphi$ is a psh weight, then $d d^{c} \varphi$ cannot charge any analytic curve. As a partial converse, any psh function with logarithmic singularities and which does not charge any analytic curves defines a psh weight.

For any psh weight $\varphi$ and any psh function $u$, the wedge product $d d^{c} u \wedge d d^{c} \varphi$ is admissible [De1], and defines a positive measure. We may hence associate to any psh function $u$ a generalized Lelong number by setting

$$
\nu_{\varphi}(u)=\lim _{r \rightarrow 0} \int_{\varphi \leq \log r} d d^{c} u \wedge d d^{c} \varphi=d d^{c} u \wedge d d^{c} \varphi\{0\} .
$$

These generalized Lelong numbers were designed to give information on the nature of the singularity of $u$ at the origin. We will use two results by Demailly.

Proposition 2.2. [De1, Chapter III, Proposition 5.12]. For any psh weight $\varphi$, and any sequence of psh functions $u_{n} \rightarrow u$ in $L_{\text {loc }}^{1}$ we have $\lim \sup \nu_{\varphi}\left(u_{n}\right) \leq \nu_{\varphi}(u)$. 
Proposition 2.3. [De1, Chapter III, Theorem 7.1]. Let $\varphi_{1}, \varphi_{2}$ be two psh weights such that $\lim \sup _{p \rightarrow 0} \varphi_{1}(p) / \varphi_{2}(p) \leq 1$. Then $\nu_{\varphi_{1}}(u) \leq \nu_{\varphi_{2}}(u)$ for any psh function $u$. In particular, equality holds when $\lim _{p \rightarrow 0} \varphi_{1}(p) / \varphi_{2}(p)=1$.

When the Monge-Ampère measure $\left(d d^{c}\right)^{2} \varphi$ is concentrated at the origin, a Jensen type formula holds. Namely, the generalized Lelong number of $u$ may be computed as follows [De1, Chapter III, Proposition 6.5]:

$$
\nu_{\varphi}(u)=\lim _{r \rightarrow 0} \frac{1}{\log r} \int_{\varphi=\log r} u d \lambda_{r}
$$

where the measure $\lambda_{r}=\left(d d^{c}\right)^{2} \max \{\varphi, \log r\}$ is supported on the set $\{\varphi=\log r\}$.

An important class of weights for which (2.9) applies is $\varphi=\log \max \left\{|\phi|^{s},|\psi|^{t}\right\}$ for holomorphic germs $\phi, \psi$ without common factor, and $s, t>0$. The measure $\lambda_{r}$ is then supported on the set $\left\{|\phi|^{s}=|\psi|^{t}=r\right\}$.

When $\varphi=\log \max \left\{|x|^{a},|y|^{b}\right\}$ we recover the Kiselman number:

$$
\begin{aligned}
\nu_{a, b}^{x, y}(u) & =d d^{c} u \wedge d d^{c} \log \max \left\{|x|^{a},|y|^{b}\right\}\{0\} \\
& =\lim _{r \rightarrow 0} \frac{1}{\log r} \int u\left(d d^{c}\right)^{2} \log \max \left\{r,|x|^{a},|y|^{b}\right\} .
\end{aligned}
$$

This follows from (2.9), (2.4) and Lemma 2.4 below.

We conclude this section with the following useful result.

Lemma 2.4. Fix local coordinates $(x, y)$ and real numbers $a, b, c, d \geq 0$ such that $a d \neq b c$. Let $(s, t)$ be the solution to as $+b t=c s+d t=1$ and assume that $s, t>0$. Then the measure $\lambda_{r}=\left(d d^{c}\right)^{2} \log \max \left\{r,|x|^{a}|y|^{b},|x|^{c}|y|^{d}\right\}$ has mass $|a d-b c|$ and is proportional to the Haar-Lebesgue measure on the torus $|x|=r^{s},|y|=r^{t}$.

Proof. The measure $\lambda_{r}$ is supported where $|x|^{a}|y|^{b}=|x|^{c}|y|^{d}=r$ and is invariant under rotations in $x$ and $y$, hence must be proportional to the HaarLebesgue measure on the above-mentioned torus. Let us prove that the mass is $|a d-b c|$. In the case $a=d=1, b=c=0$, it is a simple application of Stokes' formula that the mass equals 1 . When $a, b, c, d$ are integers, consider the monomial change of coordinates $\pi(x, y)=\left(x^{a} y^{b}, x^{c} y^{d}\right)$. The topological degree of $\pi$ is equal to $|a d-b c|$. By the change of variable formula the mass of $\left(d d^{c}\right)^{2} \log \max \{r,|x|,|y|\} \circ \pi=\left(d d^{c}\right)^{2} \log \max \left\{r,|x|^{a}|y|^{b},|x|^{c}|y|^{d}\right\}$ is $|a d-b c|$ times the mass of $\left(d d^{c}\right)^{2} \log \max \{r,|x|,|y|\}$, hence the mass of $\lambda_{r}$ equals $|a d-b c|$. When $a, b, c, d$ are rational numbers with a common denominator $q$, we use the change of variables $\pi(x, y)=\left(x^{q}, y^{q}\right)$ to reduce the proof to the preceding case. Finally we conclude in the general case by continuity.

\section{EVAlUATING QUASIMONOMIAL VALUATIONS ON PSH FUNCTIONS}

Our aim in this section is to prove the following result:

Theorem 3.1. Let $\nu \in \mathcal{V}_{\mathrm{qm}}$ be a quasimonomial valuation. There exists a unique real-valued function $T_{\nu}$ on the set of psh functions, with the following properties:

- Compatibility: $T_{\nu}(\log |\psi|)=\nu(\psi)$ for $\psi \in R$;

- Monotonicity: $T_{\nu}(u) \leq T_{\nu}(v)$ if $u \geq v+O(1)$;

- Homogeneity: $T_{\nu}(s u)=s T_{\nu}(u)$ for $s \geq 0$; 
- Tropicality: $T_{\nu}(u+v)=T_{\nu}(u)+T_{\nu}(v) ; T_{\nu} \max \{u, v\}=\min \left\{T_{\nu}(u), T_{\nu}(v)\right\}$;

- Semi-continuity: $\lim \sup T_{\nu}\left(u_{n}\right) \leq T_{\nu}(u)$ when $u_{n} \rightarrow u$ in $L_{\text {loc }}^{1}$;

- Minimality: if $T_{\nu}^{\prime}$ satisfies all the properties above, then $T_{\nu} \leq T_{\nu}^{\prime}$.

We shall then simply write $T_{\nu}(u)=\nu(u)$.

Remark 3.2. The term "tropicality" refers to the fact that the two spaces consisting of psh functions, and nonnegative real numbers, both carry a tropical semiring structure: "multiplication" is given by addition and "addition" by max / min. Thus $T_{\nu}$ is a homomorphism of semi-rings.

Remark 3.3. Note that $\nu$ may be evaluated on currents: if $S=d d^{c} u$, then we define $\nu(S)=\nu(u)$. By tropicality and monotonicity, this does not depend of the choice of $u$.

Remark 3.4. When a valuation $\nu$ is equivalent to a normalized valuation in the valuative tree, i.e. $\nu=c \nu^{\prime}$ with $c>0$ and $\nu^{\prime} \in \mathcal{V}$, then we set $\nu(u):=c \nu^{\prime}(u)$. All properties listed above remain valid.

Our approach is as follows. We give two definitions of $T_{\nu}(u)$ : first as the growth rate of $u$ in a characteristic region, then as a generalized Lelong number. Both definitions depend on a choice of representation $\nu=\nu_{\phi, t}$ and a smooth germ $x$ transverse to $\phi$. Nevertheless, we show that they give the right value on psh functions with logarithmic singularities. Using Demailly approximation we then show that the definitions of $T_{\nu}$ in fact do not depend on the choice of $\phi$ and $x$.

Throughout the analysis, we will distinguish between the monomial case $m=$ $m(\nu)=1$ and the nonmonomial case $m>1$. In the former, $T_{\nu}$ can be viewed as a Kiselman number and Theorem 3.1 translates into essentially well-known properties of Kiselman numbers. As we show, the case $m>1$ can then be reduced to the case $m=1$ through a carefully devised monomialization procedure.

For the rest of this section we fix a quasimonomial valuation $\nu \in \mathcal{V}_{\mathrm{qm}}$.

3.1. Characteristic regions. Write $\nu=\nu_{\phi, t}$ for some irreducible $\phi \in \mathfrak{m}$. Unlike the skewness $t$, the germ $\phi$ is not uniquely determined by $\nu$, but we may - and will - assume that $\phi$ has minimal multiplicity, i.e. $m(\phi)=m(\nu)=: m$. Pick $x \in \mathfrak{m}$ with $m(x)=1$ and $\nu_{x} \cdot \nu_{\phi}=1$, i.e. $\{x=0\}$ is smooth and transverse to $\{\phi=0\}$.

Definition 3.5. An open set of the form

$$
\Omega_{\phi, t, x}(r):=\left\{|x|<r,|\phi|<|x|^{m t}\right\},
$$

for small $r>0$, is called a characteristic region for $\nu$ : see Figure 2 on p. 6 .

Our goal is to show:

Proposition 3.6. If $u$ is $p s h$, then the limit

$$
\nu_{\phi, t, x}(u):=\lim _{r \rightarrow 0} \frac{1}{\log r} \sup _{\Omega_{\phi, t, x}(r)} u
$$

exists. Moreover

$$
u(q) \leq \nu_{\phi, t, x}(u) \log \|q\|+O(1)
$$

for all $q$ in $\Omega_{\phi, t, x}(r)$. 
The dependence of the characteristic region on the choices of $\phi$ and $x$ is quite weak. In fact, we will show later that the quantity $\nu_{\phi, t, x}(u)$ does not depend on these choices. In doing so, it will be important to control the volumes of the characteristic regions:

Proposition 3.7. There exists $C=C(\phi, t, x)>0$ such that

$$
C^{-1} r^{2 A} \leq \operatorname{Vol} \Omega_{\phi, t, x}(r) \leq C r^{2 A}
$$

for small $r$, where $A=A(\nu)$ is the thinness of valuation $\nu=\nu_{\phi, t}$.

For the proofs, we consider the cases $m=1$ and $m>1$ separately.

3.2. The monomial case. We first assume that $m(\nu)=1$. Then $(x, \phi)$ define local coordinates. Write $y=\phi$ for definiteness. In coordinates $(x, y)$, the valuation $\nu=\nu_{y, t}$ is monomial and the characteristic region is of the form

$$
\Omega_{\phi, t, x}(r)=\left\{|x|<r,|y|<|x|^{t}\right\},
$$

the volume of which is given by $C r^{2+2 t}$ for some constant $C>0$. This proves Proposition 3.7 in this case as $A(\nu)=1+t$.

Further, the existence of the limit in (3.2) and the inequality (3.3) both follow from the discussion of Kiselman numbers in Section 2.3. Indeed, we have

$$
\nu_{\phi, t, x}(u)=\nu_{1, t}^{x, y}(u)
$$

in this case, i.e. $\nu$ is a Kiselman number in coordinates $(x, y)$.

3.3. Monomialization. Many statements about quasimonomial valuations can be reduced to the monomial case through a procedure that we will refer to as monomialization. Since this procedure is of fundamental importance to our analysis, we discuss it in some detail.

Consider a quasimonomial valuation $\nu$ and write $\nu=\nu_{\phi, t}$ with $m(\phi)=m(\nu)=$ $m$ as above. Also pick a transverse germ $x$. We will assume that $m>1$. This implies $t>1$. Consider the approximating sequence $\nu_{\mathfrak{m}}=\nu_{0}<\nu_{1}<\cdots<\nu_{g}<\nu$ of $\nu$ as in (1.1). The valuation $\nu_{g}$ is divisorial and of the form $\nu_{\phi, t_{0}}$ for some $t_{0} \in(1, t)$. It has generic multiplicity $m$.

Let $\pi: X \rightarrow\left(\mathbf{C}^{2}, 0\right)$ be the minimal desingularization of the curve $C=\{\phi=0\}$. This can be constructed as follows. Let $p_{1}, p_{2}, \ldots$ be the sequence of infinitely nearby points associated to $C$. They are defined recursively: $p_{j+1}$ is the intersection of the strict transform of $C$ with the exceptional divisor of the blowup at $p_{j}$. We denote by $p=p_{n+1}$ the first point for which the strict transform of $\phi$ is smooth and transverse to the exceptional divisor at $p$. In suitable coordinates this strict transform is given by $\{w=0\}$, and the exceptional divisor at $p$ by $E=\{z=0\}$.

Then $\pi$ is the composition of blowups at $p_{0}, \ldots, p_{n}$. Write $J \pi$ for the Jacobian determinant of $\pi$. For $s \in[0, \infty)$, write $\mu_{s}$ for the monomial valuation sending $z$ to 1 and $w$ to $s$. Notice that $\mu_{0}=\operatorname{div}_{E}$. By [FJ1, Corollary 6.42] the divisorial valuation associated to $E$ is precisely $\nu_{g}$. In particular, $\pi_{*} \operatorname{div}_{E}=m \nu_{g}$.

Proposition 3.8. Let $m, t_{0}$ and $A_{0}$ be the generic multiplicity, skewness and thinness of $\nu_{g}$, respectively. Then the following hold:

(i) if $s \geq 0$ then $\pi_{*} \mu_{s}=m \nu_{\phi, t_{0}+m^{-2} s}$; in particular $\pi_{*} \mu_{m^{2}\left(t-t_{0}\right)}=m \nu$; 
(ii) after multiplying $z$ and $w$ by units, if necessary, we have

$$
\pi^{*} x=z^{m}, \quad \pi^{*} \phi=z^{m^{2} t_{0}} w \quad \text { and } \quad J \pi=z^{m A_{0}-1} \xi
$$

for some unit $\xi$

(iii) for any $r>0$ small enough, the contraction map $\pi$ induces a biholomorphism from the open set $\Omega_{+}=\left\{(z, w),|z|<r^{1 / m},|w|<|z|^{m^{2}\left(t-t_{0}\right)}\right\}$ onto the characteristic region $\Omega_{\phi, t, x}(r)$ defined in (3.1). See Figure 4.
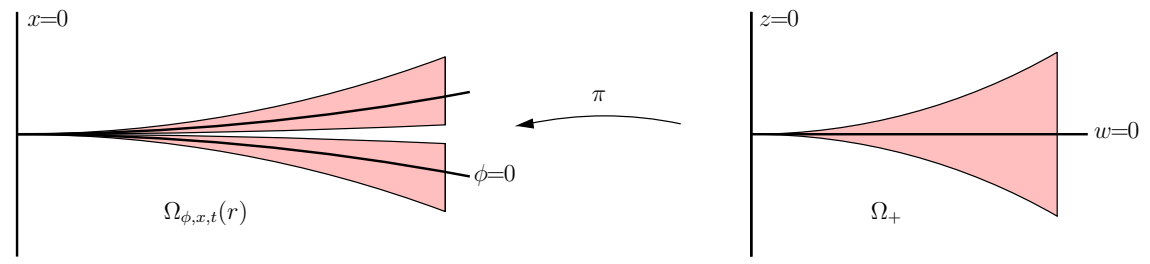

Figure 4. Monomialization. See Proposition 3.8

The contraction map $\pi$ can also be used to show that $\mathcal{V}$ has a self-similar structure: see [FJ1, Theorem 6.51, Figure 6.12].

Proof. We start by proving (ii). Since $x$ is transverse to $\phi$, the strict transform of $x$ under $\pi$ is invertible at $p$. As $\pi_{*} \mu_{0}=m \nu_{g}$ and $\nu_{g}(x)=1$, this gives $\pi^{*} x=z^{m} \eta_{1}$ for some unit $\eta_{1}$. We may assume $\eta_{1}=1$ after multiplying $z$ by a unit. Similarly, since $\nu_{g}(\phi)=m t_{0}$ and the strict transform of $\{\phi=0\}$ is $\{w=0\}$, we get $\pi^{*} \phi=z^{m^{2} t_{0}} w \eta_{2}$ for some unit $\eta_{2}$. By adjusting $w$ we may assume $\eta_{2}=1$. Finally, the formula for $J \pi$ follows from the discussion in Section 1.10.

As for (i), write $\nu_{s}=m^{-1} \pi_{*} \mu_{s}$ for $s \geq 0$. Then $\nu_{s}(x)=m^{-1} \mu_{s}\left(z^{m}\right)=1$ and $\nu_{s}(\phi)=m^{-1} \mu_{s}\left(z^{m^{2} t_{0}} w\right)=m t_{0}+m^{-1} s>m$ by (ii). In particular, since $\{x=0\}$ and $\{\phi=0\}$ are transverse, $\nu_{s}$ is normalized, i.e. $\nu_{s}(\mathfrak{m})=1$. Since $\pi$ is a morphism, the assignment $s \mapsto \nu_{s}$ is therefore a continuous map of $[0, \infty]$ into $\mathcal{V}$. If $s$ is irrational, then $\nu_{s}(\phi)=m t_{0}+m^{-1} s$ is also irrational and this implies that $\nu_{s}=\nu_{\phi, t_{0}+m^{-2} s}$ : see Section 1.4. By continuity, this must hold for all $s$, proving (i).

Let us finally prove the analytic statement (iii). Fix $r_{0}$ such that $\pi$ is welldefined and $\xi$ invertible in the region $\Omega_{+}$for $r \leq r_{0}$ and consider $r<r_{0}$. It follows from (ii) that $\pi$ is an injective map of $\Omega_{+}$into $\Omega_{\phi, t, x}(r)$. Let us prove that it is surjective.

Fix $x$ with $|x|<r$ and let $A_{x}$ be the set $\left\{y ;|\phi(x, y)|<|x|^{m t}\right\}$. This is a possibly disconnected open subset of $\mathbf{C}$. Let $\omega$ be one of its components. The proof is complete if we can find $z$ with $z^{m}=x$ such that $\pi_{z}$, defined by $\pi(z, w)=$ $\left(z^{m}, \pi_{z}(w)\right)$, maps the disk $\Delta=\left\{w ;|w|<r^{1 / m}\right\}$ onto $\omega$.

Notice that $y \rightarrow|\phi(x, y)|^{2}$, being harmonic and non constant, is an open mapping, hence $\partial \omega$ is equal to $\left\{y ;|\phi(x, y)|=|x|^{m t}\right\}$. Moreover, $\omega$ contains a point $y_{0}$ (possibly several) such that $\phi\left(x, y_{0}\right)=0$. Since $\{w=0\}$ is the strict transform of $\{\phi=0\}$ under $\pi$, there exists $z$ such that $\pi(z, 0)=\left(x, y_{0}\right)$. Then $\pi_{z}(0)=y_{0}$ so since $\Delta$ is connected we have $\pi_{z}(\Delta) \subset \omega$. As the map $\pi_{z}$ is open, its image 
$\pi_{z}(\Delta)$ is open in $\omega$. Pick $q_{n} \in \pi_{z}(\Delta)$ converging to $q \in \omega$, and write $q_{n}=\pi_{z}\left(p_{n}\right)$, $p_{n} \in \Delta$. Extract a subsequence $p_{n} \rightarrow p \in \bar{\Delta}$. By continuity $\pi_{z}(p)=q$. But (ii) shows that $\pi_{z}(\partial \Delta) \subset \partial \omega$, hence $p \in \Delta$, and $\pi_{z}(\Delta)$ is closed in $\omega$. The open set $\omega$ being connected we conclude that $\pi_{z}(\Delta)=\omega$, which completes the proof.

We are now ready to prove the existence of $\nu_{\phi, t, x}(u)$ and the volume estimate in Proposition 3.7 in the nonmonomial case $m>1$.

Proof of Proposition 3.7. Write $s=m^{2}\left(t-t_{0}\right)$. By Proposition 3.8 we have $|J \pi(z, w)| \simeq|z|^{m A_{0}-1}$ in $\Omega_{+}$if $r$ is small enough. By the change of variables formula, this yields

$$
\operatorname{Vol} \Omega_{\phi, t, x}(r)=\int_{\Omega_{+}}|J \pi|^{2} \simeq \int_{|z|<r \frac{1}{m},|w|<|z|^{s}}|z|^{2 m A_{0}-2} \simeq r^{m^{-1}\left(2+2 s+2 m A_{0}-2\right)},
$$

which completes the proof, since $A(\nu)=A_{0}+m\left(t-t_{0}\right)=A_{0}+m^{-1} s$.

Proof of Proposition 3.6. Again we use Proposition 3.8, which gives

$$
\frac{1}{\log r} \sup _{\Omega_{\phi, t, x}(r)} u=\frac{1}{\log r} \sup \left\{\pi^{*} u ;|z|<r^{\frac{1}{m}},|w|<|z|^{s}\right\} .
$$

for small $r$. Hence $\nu_{\phi, t, x}(u)$ is well-defined and we have

$$
\nu_{\phi, t, x}(u)=m^{-1} \nu_{1, s}^{z, w}\left(\pi^{*} u\right),
$$

where $\nu^{z, w}$ denotes the Kiselman number in coordinates $(z, w)$. Notice that (3.6) exhibits $\nu_{\phi, t, x}$ as the pushforward of a Kiselman number.

As for (3.3), pick $q=(x, y) \in \Omega_{\phi, t, x}(r)$ and write $q=\pi\left(q^{\prime}\right)$ where $q^{\prime}=(z, w)$. Let us first assume that $s \geq 1$. Then $\left\|q^{\prime}\right\| \simeq|z|=|x|^{1 / m} \simeq\|q\|^{1 / m}$ so (2.5) implies

$$
u(q)=\left(\pi^{*} u\right)\left(q^{\prime}\right) \leq \nu_{1, s}^{z, w}\left(\pi^{*} u\right) \log \left\|q^{\prime}\right\|+O(1)=\nu_{\phi, t, x}(u) \log \|q\|+O(1) .
$$

If instead $s<1$, then $\left\|q^{\prime}\right\| \simeq|w|=|\phi| /|z|^{m^{2} t_{0}} \leq|x|^{s / m} \simeq|q|^{s / m}$, so

$$
\begin{aligned}
u(q) & \leq \frac{1}{s} \nu_{1, s}^{z, w}\left(\pi^{*} u\right) \log \left\|q^{\prime}\right\|+O(1) \leq \frac{m}{s} \nu_{\phi, t, x}(u) \frac{s}{m} \log \|q\|+O(1) \\
& =\nu_{\phi, t, x}(u) \log \|q\|+O(1),
\end{aligned}
$$

which concludes the proof.

3.4. Analytic definition. We have defined $\nu_{\phi, t, x}$ as a growth rate in the characteristic region. Let us give a more analytic definition.

Proposition 3.9. Given $\phi, t$ and $x$ as above, we have

$$
\nu_{\phi, t, x}(u)=d d^{c} u \wedge d d^{c} \log \max \left\{|x|^{t},|\phi|^{1 / m}\right\}\{0\}
$$

for any psh function $u$. Thus $\nu_{\phi, t, x}(u)$ can be viewed as the generalized Lelong number $\nu_{\varphi}(u)$ with weight $\varphi=\log \max \left\{|x|^{t},|\phi|^{1 / m}\right\}$.

Proof. Write $\nu_{\phi, t, x}^{\text {an }}(u)$ for the right hand side of (3.7).

In the monomial case $m=m(\nu)=1,(3.7)$ reduces to a well-known statement about Kiselman numbers by putting $y=\phi$ : see (3.4) and (2.10). 
When $m>1$ then monomialization as in Proposition 3.8 gives:

$$
\begin{aligned}
\nu_{t, \phi, x}^{\text {an }}(u) & =\lim _{r \rightarrow 0} \frac{1}{\log r} \int u\left(d d^{c}\right)^{2} \log \max \left\{r,|x|^{t},|\phi|^{1 / m}\right\} \\
& =\lim _{r \rightarrow 0} \frac{1}{m t \log r} \int\left(\pi^{*} u\right)\left(d d^{c}\right)^{2} \log \max \left\{r^{m t},|z|^{m t},|w|^{1 / m}|z|^{m t_{0}}\right\} \\
& =\lim _{r \rightarrow 0} \frac{1}{m \log r} \int\left(\pi^{*} u\right)\left(d d^{c}\right)^{2} \log \max \left\{r,|z|,|w|^{s}\right\} \\
& =m^{-1} \nu_{1, s}^{z, w}\left(\pi^{*} u\right),
\end{aligned}
$$

where $s=m^{2}\left(t-t_{0}\right)$. Here the first equality is a consequence of (2.9), the second follows from the change of variables formula and from replacing $r$ by $r^{m t}$. In the third equality we have used Lemma 2.4. The last line follows from (2.10). Thus (3.6) implies that $\nu_{t, \phi, x}^{\text {an }}(u)=\nu_{\phi, t, x}(u)$.

3.5. Monotonicity, tropicality and homogeneity. It is immediate from (3.2) that if $u$ and $v$ are psh functions with $u \geq v+O(1)$, then $\nu_{\phi, t, x}(u) \leq \nu_{\phi, t, x}(v)$. Moreover, we have

Proposition 3.10. Given $\phi, t$ and $x$ as above, we have

$$
\nu(s u+t v)=s \nu(u)+t \nu(v) \quad \text { and } \quad \nu(\max \{u, v\})=\min \{\nu(u), \nu(v)\},
$$

for any psh functions $u, v$ and any constants $s, t \geq 0$. Here $\nu=\nu_{\phi, t, x}$.

Proof. The first equality is an immediate consequence of Proposition 3.9. As $u \leq \max \{u, v\},(3.2)$ immediately implies $\nu(\max \{u, v\}) \leq \nu(u)$. By symmetry we obtain $\nu(\max \{u, v\}) \leq \min \{\nu(u), \nu(v)\}$. For the reverse inequality we use (3.3): in $\Omega_{\phi, t, x}(r)$ we have $u(q) \leq \nu(u) \log \|q\|+O(1)$ and $v(q) \leq \nu(v) \log \|q\|+O(1)$. Thus $\max \{u, v\}(q) \leq \min \{\nu(u), \nu(v)\} \log \|q\|+O(1)$, so (3.2) immediately gives $\nu(\max \{u, v\}) \geq \min \{\nu(u), \nu(v)\}$.

3.6. Compatibility. Next we show that independently of any choice of $\phi$ and $x$, the definition of $\nu(u)$ agrees with the algebraic definition when $u$ has logarithmic singularities, in the following sense.

Proposition 3.11. Let $u=c \log \left(\sum_{1}^{n}\left|\psi_{i}\right|\right)+O(1), c>0, \psi_{i} \in R$, be a psh function with logarithmic singularities. Then

$$
\nu_{\phi, t, x}(u)=c \min _{i} \nu\left(\psi_{i}\right) .
$$

Here $\nu\left(\psi_{i}\right)$ denotes the value of $\nu$ on the holomorphic germ $\psi_{i} \in R$.

In particular, the definition of $\nu(u)$ is independent of the choices of $\phi$ and $x$ when $u$ has logarithmic singularities.

Proof. We may assume $c=1$ and that the $O(1)$ term is absent. Moreover, $\min \log \left|\psi_{i}\right| \leq \log \sum_{1}^{n}\left|\psi_{i}\right| \leq \min \log \left|\psi_{i}\right|+\log n, \operatorname{so} \nu\left(\log \sum\left|\psi_{i}\right|\right)=\min \nu\left(\log \left|\psi_{i}\right|\right)$ as follows from (3.3). We can hence suppose $u=\log |\psi|$ for $\psi \in R$.

First consider the monomial case $m=m(\nu)=1$. Write $y=\phi$ for definiteness. Then $\psi$ can be viewed as a power series in coordinates $(x, y)$, say $\psi=\sum a_{k l} x^{k} y^{l}$. By definition $\nu(\psi)=\min \left\{k+t l ; a_{k l} \neq 0\right\}=: \alpha$. The characteristic region is given 
by $\Omega_{\phi, t, x}(r)=\left\{|x|<r,|y|<r^{t}\right\}$ and it is elementary to see that $\sup |\psi| \simeq r^{\alpha}$ on $\Omega_{\phi, t, x}(r)$. Therefore, $\nu_{\phi, t, x}(\log |\psi|)=\alpha=\nu(\psi)$.

When $m>1$, we monomialize, using Proposition 3.8. Write $s=m^{2}\left(t-t_{0}\right)$. Then $\nu=m^{-1} \pi_{*} \mu_{s}$ so by (3.6) and the monomial case above, we get

$$
\nu_{\phi, t, x}(\log |\psi|)=m^{-1} \nu_{1, s}^{z, w}\left(\log \left|\pi^{*} \psi\right|\right)=m^{-1} \mu_{s}\left(\pi^{*} \psi\right)=\nu(\psi) .
$$

This concludes the proof.

3.7. Approximation. To show that the definition of $\nu(u)$, for a general psh function $u$, is independent of all choices made, we use Demailly approximation.

Proposition 3.12. For any psh function $u$, there exists a sequence $u_{n}$ of psh functions with logarithmic singularities such that $u_{n} \rightarrow u$ in $L_{\mathrm{loc}}^{1}$ and

$$
0 \leq \nu_{\phi, t, x}(u)-\nu_{\phi, t, x}\left(u_{n}\right) \leq \frac{A}{n}
$$

for any choice of $\phi$ and $x$. Here $A=A(\nu)$ is the thinness of $\nu=\nu_{\phi, t}$.

Remark 3.13. The estimate (3.9) for Lelong numbers (i.e. $\nu=\nu_{\mathfrak{m}}$ ) is due to Demailly (see [DK, Theorem 4.2]). The extension to Kiselman numbers (i.e. $m(\nu)=1$ ) was proved independently by Rashkovskii [Ra, Theorem 3] and the authors [FJ3, Lemma 2.4].

Proof. We choose $u_{n}$ as in Section 2.2, i.e. $u_{n}=n^{-1} \sup \log |f|$ where the supremum is over holomorphic functions $f$ such that $\int|f|^{2} \exp (-2 n u) \leq 1$ over a fixed neighborhood of the origin.

By (2.1), $u_{n} \geq u-C$ for some constant $C>0$, hence $\nu_{\phi, t, x}\left(u_{n}\right) \leq \nu_{\phi, t, x}(u)$. For the other inequality, consider the open set

$$
\Omega^{\prime}(r)=\Omega_{\phi, t, x}^{\prime}(r)=\left\{\frac{1}{2} r<|x|<r, \frac{1}{2}|x|^{m t}<|\phi|<|x|^{m t}\right\} .
$$

With minor modifications, the proof of Proposition 3.7 shows that $\operatorname{Vol} \Omega^{\prime}(r) \simeq$ $r^{2 A(\nu)}$ and by the maximum principle $(\log r)^{-1} \sup _{\Omega^{\prime}(r)} v=(\log r)^{-1} \sup _{\Omega(r)} v \rightarrow$ $\nu_{\phi, t, x}(v)$ as $r \rightarrow 0$, for any psh function $v$. We need the following estimate:

Lemma 3.14. For any $\varepsilon \in(0,1)$ there exists $C_{\varepsilon}>1$ such that

$$
C_{\varepsilon} \sup _{\Omega^{\prime}(\varepsilon r)} v \leq \frac{1}{\operatorname{Vol} \Omega^{\prime}(r)} \int_{\Omega^{\prime}(r)} v
$$

for small $r$ and any psh function $v \leq 0$. Moreover, $C_{\varepsilon} \rightarrow 1$ as $\varepsilon \rightarrow 0$.

We apply this lemma to $v=\log |f|^{2}$, where $\int|f|^{2} \exp (-2 n u) \leq 1$. Using the concavity of the logarithm we get

$$
\begin{gathered}
C_{\varepsilon} \sup _{\Omega^{\prime}(\varepsilon r)} \log |f|^{2} \leq \frac{1}{\operatorname{Vol} \Omega^{\prime}(r)} \int_{\Omega^{\prime}(r)} \log |f|^{2} \leq \log \left[\frac{1}{\operatorname{Vol} \Omega^{\prime}(r)} \int_{\Omega^{\prime}(r)}|f|^{2}\right] \leq \\
\quad \leq \log \left[\frac{1}{\operatorname{Vol} \Omega^{\prime}(r)} \sup _{\Omega^{\prime}(r)} e^{2 n u} \int_{\Omega^{\prime}(r)}|f|^{2} e^{-2 n u}\right]=2 n \sup _{\Omega^{\prime}(r)} u-\log \operatorname{Vol} \Omega^{\prime}(r) .
\end{gathered}
$$


As $\log \operatorname{Vol} \Omega^{\prime}(r)=2 A(\nu) \log r+O(1)$ we get $C_{\varepsilon} \nu_{\phi, t, x}(\log |f|) \geq n \nu_{\phi, t, x}(u)-A(\nu)$ by letting $r \rightarrow 0$. Letting $\varepsilon \rightarrow 0$ yields $\nu_{\phi, t, x}(\log |f|) \geq n \nu_{\phi, t, x}(u)-A(\nu)$. Using $u_{n}=n^{-1} \sup \log |f|$, we conclude $\nu_{\phi, t, x}\left(u_{n}\right) \geq \nu_{\phi, t, x}(u)-A(\nu) / n$.

Proof of Lemma 3.14. We only prove this result in the case $m>1$, the monomial one $(m=1)$ being easier. As usual we make use of the monomialization procedure in Proposition 3.8. Write

$$
\mathcal{D}(r)=\left\{\left(\frac{1}{2} r\right)^{\frac{1}{m}}<|z|<r^{\frac{1}{m}}, \frac{1}{2}|z|^{s}<|w|<|z|^{s}\right\} .
$$

Then $\pi$ is a biholomorphism from $\mathcal{D}(r)$ onto $\Omega^{\prime}(r)$. Let $D(r)=\{(|z|,|w|) ;(z, w) \in$ $\mathcal{D}(r)\} \subset \mathbf{R}^{2}$. For $\rho=\left(\rho_{1}, \rho_{2}\right) \in D(r)$ let $T_{\rho}$ be the torus $\left\{|z|=\rho_{1},|w|=\rho_{2}\right\} \subset$ $\mathcal{D}(r)$. For any $\varepsilon \in(0,1)$ and any $\rho \in D(r)$, Lemma 2.1 and the maximum principle imply

$$
C_{\varepsilon} \sup _{\Omega^{\prime}(\varepsilon r)} v=C_{\varepsilon} \sup _{\mathcal{D}(\varepsilon r)} \pi^{*} v \leq \int_{T_{\rho}} \pi^{*} v
$$

where the integral is with respect to Lebesgue measure of mass one on the torus. Note that we can take $C_{\varepsilon}=(1+\varepsilon)^{4} /\left(1-\varepsilon^{2}\right)$ hence $C_{\varepsilon} \rightarrow 1$ when $\varepsilon \rightarrow 0$.

The critical set of $\pi$ is equal to $\{z=0\}$. We can thus write $C^{\prime}|z|^{m A_{0}-1} \geq$ $|J \pi| \geq C|z|^{m A_{0}-1}$ on $\mathcal{D}(r)$, for some constants $C=C(r), C^{\prime}=C^{\prime}(r)>0$, with $\lim _{r \rightarrow 0} C(r) / C^{\prime}(r)=1$. We have

$$
\begin{gathered}
\int_{\Omega^{\prime}(r)} v=\int_{\mathcal{D}(r)}|J \pi|^{2} \pi^{*} v=\int_{\rho \in D(r)}\left[\int_{T_{\rho}}|J \pi|^{2} \pi^{*} v\right] \rho_{1} \rho_{2} d \rho_{1} d \rho_{2} \\
\geq \int_{\rho \in D(r)}\left[\int_{T_{\rho}} C^{2} \rho_{1}^{2\left(m A_{0}-1\right)} \pi^{*} v\right] \rho_{1} \rho_{2} d \rho_{1} d \rho_{2} \\
\geq\left(C / C^{\prime}\right)^{2} C_{\varepsilon} \int_{\rho \in D(r)}\left[\int_{T_{\rho}}|J \pi|^{2} \sup _{\mathcal{D}(\varepsilon r)} \pi^{*} v\right] \rho_{1} \rho_{2} d \rho_{1} d \rho_{2}=C_{\varepsilon}^{\prime} \operatorname{Vol} \Omega^{\prime}(r) \sup _{\Omega^{\prime}(\varepsilon r)} v
\end{gathered}
$$

with $C_{\varepsilon}^{\prime} \rightarrow 1$ as $\varepsilon \rightarrow 0$.

3.8. Proof of Theorem 3.1. Let us summarize our construction. Fix a quasimonomial valuation $\nu$. Write $\nu=\nu_{\phi, t}$ with $m(\phi)=m(\nu)=: m$ and pick a holomorphic germ $x$ such that $\{x=0\}$ is smooth and transverse to $\{\phi=0\}$. We defined $T_{\nu}$ in two equivalent ways: as a growth rate on a characteristic region (Proposition 3.6) and as a generalized Lelong number (Proposition 3.9). These definitions a priori depended on the choices of $\phi$ and $x$ but we showed independence of these choices when restricted to psh functions with logarithmic singularities (Proposition 3.11). Using Demailly approximation (Proposition 3.12), this was extended to arbitrary psh functions. Monotonicity, homogeneity and tropicality were proved in Section 3.5. Semi-continuity is an immediate consequence of the analytic definition of $T_{\nu}$. Compatibility was proved in Proposition 3.11. Finally, let us prove minimality (and hence uniqueness). By compatibility we have $T_{\nu}^{\prime}(u)=T_{\nu}(u)$ when $u=\log |\phi|, \phi \in R$. By Proposition 3.11, tropicality, and monotonicity, the same result holds when $u$ has logarithmic singularities. Semi-continuity together with 
Proposition 3.12 then shows that $T_{\nu}^{\prime}(u) \geq T_{\nu}(u)$ for all $u$. This concludes the proof of Theorem 3.1.

\section{Further properties}

In this section we give further information on the pairing $(\nu, u) \mapsto \nu(u)$.

4.1. Geometric interpretation. Let us prove the following geometric interpretation of $\nu(u)$ in case $\nu$ is divisorial.

Proposition 4.1. Suppose $\nu \in \mathcal{V}$ is a divisorial valuation, associated to an exceptional component $E$ of a modification $\pi$ above the origin. Write $\nu=\nu_{E}=$ $b^{-1} \pi_{*} \operatorname{div}_{E}$, with $b$ the generic multiplicity of $\nu$.

Then for any psh function $u, \nu(u)$ is equal to $1 / b$ times the Lelong number of $\pi^{*} u:=u \circ \pi$ taken at a generic point of $E$.

Proof. By Siu's theorem, the Lelong number of $\pi^{*} u$ at all points $p \in E$ except for countably many is the same, equal to some constant $M \geq 0$. Pick a point $p \in E$ in the regular set of $\pi^{-1}(0)$ such that $\nu^{\mathrm{L}}\left(\pi^{*} u, p\right)=M$. Choose a smooth curve $V$ cutting $E$ transversely at $p$. We let $\phi \in \mathfrak{m}$ be an equation defining $\pi(V)$. By Section 1.10, $\nu_{\phi} \geq \nu$ hence $\nu=\nu_{\phi, t}$ for some $t$. Fix local coordinates $(z, w)$ at $p$, such that $E=\{z=0\}$, and $V=\{w=0\}$, and a coordinate axis $x$ at the origin in $\mathbf{C}^{2}$ such that Proposition 3.8 (iii) applies: the map $\pi$ gives a biholomorphism between $\Delta_{r^{1 / b}} \times \Delta_{1}$ and $\Omega_{\phi, x, t}(r)=\left\{|x|<r,|\phi|<|x|^{b t}\right\}$ (note that in Proposition 3.8 the generic multiplicity of $\nu$ is denoted by $m$ ). We hence

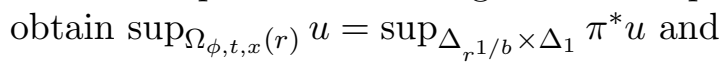

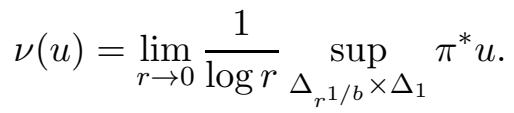

As $\Delta_{r^{1 / b}} \times \Delta_{r^{1 / b}} \subset \Delta_{r^{1 / b}} \times \Delta_{1}$ we get $b \times \nu(u) \leq \nu^{\mathrm{L}}\left(\pi^{*} u, p\right)=M$.

It is also a consequence of Siu's theorem that the function $\pi^{*} u-M \log |z|$ is psh. By the maximum principle, the supremum of $\pi^{*} u-M \log |z|$ on $\Delta_{r^{1 / b}} \times \Delta_{1}$ is attained on $\partial \Delta_{r^{1 / b}} \times \Delta_{1}$, hence

$$
\begin{aligned}
0 \leq \lim _{r \rightarrow 0} & \frac{1}{\log r} \sup \left\{\pi^{*} u-M \log |z| ;|z|<r^{1 / b},|w|<1\right\}= \\
& =\lim _{r \rightarrow 0} \frac{1}{\log r} \sup \left\{\pi^{*} u-\frac{M}{b} \log r ;|z|=r^{1 / b},|w|<1\right\}= \\
= & -\frac{M}{b}+\lim _{r \rightarrow 0} \frac{1}{\log r} \sup \left\{\pi^{*} u ;|z|<r^{1 / b},|w|<1\right\}=-\frac{M}{b}+\nu(u) \leq 0
\end{aligned}
$$

We conclude that $b \times \nu(u)=M$.

4.2. Action by holomorphic maps. Next we prove that the evaluation map $(\nu, u) \rightarrow \nu(u)$ behaves well under the action of a holomorphic map, a fact relevant to the study of the action of holomorphic maps on the valuative tree [FJ4]. 
Proposition 4.2. Suppose $f:\left(\mathbf{C}^{2}, 0\right) \rightarrow\left(\mathbf{C}^{2}, 0\right)$ is a holomorphic germ whose Jacobian determinant does not vanish identically. Then for any plurisubharmonic function $u$ and any quasimonomial valuation $\nu$, we have

$$
\nu\left(f^{*} u\right)=\left(f_{*} \nu\right)(u)
$$

where $f^{*} u:=u \circ f$, and $f_{*} \nu$ is the valuation defined by $\left(f_{*} \nu\right)(\phi)=\nu\left(f^{*} \phi\right)$ for $\phi \in R$.

Note that in general $f_{*} \nu$ is no longer normalized: there is no reason why $c(\nu):=$ $\left(f_{*} \nu\right)(\mathfrak{m})=1$. It is however equivalent to a unique normalized valuation $f_{\bullet} \nu \in \mathcal{V}$, and we can set $\left(f_{*} \nu\right)(u):=c(\nu) \times\left(f_{\bullet} \nu\right)(u)$ for any psh function $u$.

Proof. If $u=c \log \sum_{1}^{n}\left|\phi_{i}\right|^{2}$, where $c>0$ and $\phi_{i} \in R$ are holomorphic germs, then (4.1) is an immediate consequence of Proposition 3.11. Thus (4.1) holds when $u$ has logarithmic singularities in view of Proposition 3.10.

Now consider a general plurisubharmonic function $u$ and approximate it by psh functions $u_{n}$ with logarithmic singularities as in Proposition 3.12. Recall that $u_{n}=n^{-1} \sup \log |h|$ where $h$ ranges over all holomorphic functions such that $\int_{B}|h|^{2} \exp (-2 n u) \leq 1$, and $B$ is a fixed ball centered at 0 . Define in the same way $\tilde{u}_{n}:=n^{-1} \sup \log |\tilde{h}|$ where $\tilde{h}$ ranges over all holomorphic functions such that $\int_{B}|\tilde{h}|^{2} \exp \left(-2 n f^{*} u\right) \leq 1$. This sequence approximates $f^{*} u$. By choosing suitable coordinates at the source space, we may assume that $f(B) \subset B$. Denote by $J f$ the Jacobian determinant of $f$. The change of variables formula yields

$$
\int_{B}\left|f^{*} h \cdot J f\right|^{2} \exp \left(-2 n f^{*} u\right) \leq e \int_{f(B)}|h|^{2} \exp (-2 n u) \leq e \int_{B}|h|^{2} \exp (-2 n u),
$$

where $e$ denotes the topological degree of $f$, i.e. the cardinality of a generic fiber of $f$. We thus infer $\tilde{u}_{n} \geq f^{*} u_{n}+n^{-1} \log |J f / \sqrt{e}|$ for all $n$. Using Proposition 3.12 we conclude $\nu\left(f^{*} u\right)=\lim \nu\left(\tilde{u}_{n}\right) \leq \liminf \nu\left(f^{*} u_{n}\right)$. On the other hand, $f^{*} u_{n} \rightarrow f^{*} u$ in $L_{\text {loc }}^{1}$, so by semi-continuity $\nu\left(f^{*} u\right) \geq \lim \sup \nu\left(f^{*} u_{n}\right)$. This shows that $\nu\left(f^{*} u\right)=$ $\lim \nu\left(f^{*} u_{n}\right)$, so

$$
\left(f_{*} \nu\right)(u)=\lim _{n \rightarrow \infty}\left(f_{*} \nu\right)\left(u_{n}\right)=\lim _{n \rightarrow \infty} \nu\left(f^{*} u_{n}\right)=\nu\left(f^{*} u\right),
$$

which concludes the proof.

4.3. Estimates. The characteristic regions we used to defined $\nu(u)$ can be replaced by other semi-analytic regions. For constants $C_{1}, C_{2}, C_{3}, C_{4}>0$ introduce $\mathcal{A}_{\phi, t, x, C}(r)=\left\{C_{1} r<|x|<C_{2} r ; C_{3}|x|^{m t}<|\phi|<C_{4}|x|^{m t}\right\}$. With these regions we can strengthen Proposition 3.6 as follows.

Proposition 4.3. For any psh function $u$ and any $\nu \in \mathcal{V}_{\mathrm{qm}}$ :

$$
\begin{aligned}
& \nu(u)=\lim _{r \rightarrow 0} \frac{1}{\log r} \sup _{\mathcal{A}_{\phi, t, x, C}(r)} u \quad \text { and } \\
& u(q) \leq \nu(u) \log \|q\|+O(1) \quad \text { for all } q \in \mathcal{A}_{\phi, t, x, C}(r) .
\end{aligned}
$$

When $u$ has logarithmic singularities, we may pick $C=C(u)$ such that

$$
u(q) \geq \nu_{\phi, t, x}(u) \log \|q\|+O(1) \quad \text { for all } q \in \mathcal{A}_{\phi, t, x, C}(r) .
$$


Proof. The first two assertions are immediate consequences of the maximum principle. For the last assertion, we may suppose $u=\log |\psi|, \psi \in \mathfrak{m}$ and $\nu$ is a monomial valuation sending $x$ to 1 and $y$ to $t$. Write $\psi(x, y)=\sum a_{k l} x^{k} y^{l}$ and $\nu=\nu(\psi)$. Then $\left|\sum_{k+t l>\nu} a_{k l} x^{k} y^{l}\right| \leq$ Const $\cdot r^{\nu+\varepsilon}$ for some $\varepsilon>0$. The set of $(k, l)$ such that $k+l t=\nu$ and $a_{k l} \neq 0$ is non-empty and finite. We denote by $\left(k_{0}, l_{0}\right)$ the element of this set with maximal first component (hence minimal second component), and we have $\left|a_{k_{0} l_{0}} x^{k_{0}} y^{l_{0}}\right| \geq\left|a_{k_{0} l_{0}}\right| C_{1}^{k_{0}} C_{3}^{l_{0}} r^{\nu}$. For the other $(k, l)$ with $k+l t=\nu$, we have $\left|a_{k l} x^{k} y^{l}\right| \leq\left|a_{k l}\right| C_{2}^{k} C_{4}^{l} r^{\nu}$. Now choose $C_{2}>C_{1} \gg 1$ large but sufficiently close to each other, and $C_{3}<C_{4} \ll 1$ small. With these choices of $C_{i}$ 's, we have $\left|\sum_{k+l t=\nu} a_{k l} x^{k} y^{l}\right| \geq\left|a_{k_{0} l_{0}} x^{k_{0}} y^{l_{0}}\right|-\sum_{k+l t=\nu,(k, l) \neq\left(k_{0}, l_{0}\right)}\left|a_{k l} x^{k} y^{l}\right| \geq$ Const $\cdot r^{\nu}$. Therefore, $|\psi| \geq$ Const $\cdot r^{\nu}$. This completes the proof.

\section{Potential theory on trees}

The next step in our approach is to analyze, for a fixed psh function $u$, the function $\nu \mapsto \nu(u)$ on the tree $\mathcal{V}_{\mathrm{qm}}$ of quasimonomial valuations.

As we showed in [FJ1, Chapter 7], there is a general correspondence between positive measures on trees endowed with a parameterization and certain functions called tree potentials. In this section, we review briefly this correspondence in the case of the valuative tree $\mathcal{V}$ parameterized by skewness, referring to [FJ1] for the proofs. Later we shall show that the function $\nu \mapsto \nu(u)$ is a tree potential.

5.1. Borel measures. (See [FJ1, Section 7.3].) We equip $\mathcal{V}$ with the Borel $\sigma$ algebra generated by the weak topology (see Section 1.5). We let $\mathcal{M}$ be the set of positive $^{4}$ Borel measures ${ }^{5}$ on $\mathcal{V}$, that is, continuous positive linear functionals on the set of continuous real valued functions on $\mathcal{V}$. The space $\mathcal{M}$ also carries a weak topology: $\rho_{k} \rightarrow \rho$ iff $\int \varphi d \rho_{k} \rightarrow \int \varphi d \rho$ for all $\varphi$. The subset of probability measures is compact. We identify $\nu \in \mathcal{V}$ with the corresponding point mass $\delta_{\nu} \in \mathcal{M}$.

A subtree $\mathcal{T}$ of $\mathcal{V}$ is a subset of $\mathcal{V}$ such that $\mu \in \mathcal{T}$ and $\nu \leq \mu$ imply $\nu \in \mathcal{T}$. It is a finite subtree if it has finitely many ends. The weak topology on $\mathcal{V}$ is the weakest topology restricting to the usual topology on any finite subtree.

While the valuative tree has ample branching, the support of a measure is always much thinner:

Lemma 5.1. The support of any positive measure $\rho \in \mathcal{M}$ is contained in the closure of a countable union of finite subtrees.

Proof. We may suppose that $\rho$ has mass 1 . Consider the decreasing function $f=f_{\rho}: \mathcal{V} \rightarrow[0,1]$ defined by $f(\nu)=\rho\{\mu \geq \nu\}$. The support of $\rho$ is included in the closure of the union of the trees $\mathcal{T}_{n}:=\left\{f \geq n^{-1}\right\}, n \geq 1$. By construction the tree $\mathcal{T}_{n}$ has at most $n+1$ ends.

Remark 5.2. Any positive measure is uniquely determined by its values on the sets $U(\vec{v})$ : see [FJ1, Lemma 7.18]. This is a nontrivial assertion, despite the fact that the weak topology is by definition generated by the sets $U(\vec{v})$.

\footnotetext{
${ }^{4}$ Note that in [FJ1], $\mathcal{M}$ denotes the set of complex Borel measures.

${ }^{5}$ Every positive finite measure on $\mathcal{V}$ is a Radon measure by [FJ1, Proposition 7.14].
} 
5.2. Tree potentials. (See [FJ1, Section 7.9]). We now describe the class of functions on $\mathcal{V}_{\mathrm{qm}}$ that model the behavior of tree transforms of psh functions.

Consider a function $g: \mathcal{V}_{\mathrm{qm}} \rightarrow \mathbf{R}$, and pick a tangent vector $\vec{v}$ at $\nu \in \mathcal{V}_{\mathrm{qm}}$. We define the derivative of $g$ along $\vec{v}$ (when it exists) by

$$
D_{\vec{v}} g=\lim \frac{g(\nu)-g(\mu)}{|\alpha(\nu)-\alpha(\mu)|} \text { when } \mu \text { tends to } \nu \text { along } \vec{v} \text {. }
$$

Here a sequence $\mu_{k}$ tends to $\nu$ along $\vec{v}$ iff $\mu_{k} \in U(\vec{v})$, and the segments $\left[\mu_{k}, \nu[\right.$ form a decreasing sequence with empty intersection. This is stronger than saying that $\mu_{k} \rightarrow \nu$ and $\mu_{k} \in U(\vec{v})$ for all $k$.

Note that if $g$ is increasing on $\mathcal{V}$, and the derivative of $g$ along $\vec{v}$ is well defined, then $D_{\vec{v}} g \geq 0$ when $\vec{v}$ is not represented by $\nu_{\mathfrak{m}}$, and $D_{\vec{v}} g \leq 0$ otherwise.

Definition 5.3. A function $g: \mathcal{V}_{\mathrm{qm}} \rightarrow \mathbf{R}$ is called a tree potential on $\mathcal{V}$, if the following conditions are satisfied:

(P1) $g$ is nonnegative, increasing, and concave along totally ordered segments;

(P2) if $\nu \neq \nu_{\mathfrak{m}}$, then $\sum_{\vec{v} \in T \nu} D_{\vec{v}} g \leq 0$

(P3) $\sum_{\vec{v} \in T \nu_{\mathfrak{m}}} D_{\vec{v}} g \leq g\left(\nu_{\mathfrak{m}}\right)$.

We denote by $\mathcal{P}$ the set of all tree potentials on $\mathcal{V} .^{6}$

If $\nu_{\star}$ is an end in $\mathcal{V}$, then (P1) allows us to define $g\left(\nu_{\star}\right) \in \overline{\mathbf{R}}_{+}$as well as $D_{\vec{v}} g$, where $\vec{v}$ is the unique tangent vector at $\nu_{\star}$.

As the tangent space is uncountable at any divisorial valuation, conditions (P2) and (P3) are quite strong. Indeed, if $\nu$ is divisorial, then for all but countably many $\vec{v} \in T \nu$ we must have $D_{\vec{v}} g=0$, which by (P1) implies that $g \equiv g(\nu)$ on $U(\vec{v})$ if $\vec{v}$ is not represented by $\nu_{\mathfrak{m}}$. Along these lines, we can show

Lemma 5.4. Suppose $g$ is a tree potential. Then its support

$$
\operatorname{supp} g:=\left\{\nu \in \mathcal{V}_{\mathrm{qm}} \mid g \text { not locally constant at } \nu\right\},
$$

is contained in the closure of a countable tree.

Proof. After multiplication by a constant we may assume that $g\left(\nu_{\mathfrak{m}}\right) \leq 1$. For $n \geq 1$, let $\mathcal{T}_{n}$ be the set consisting of $\nu_{\mathfrak{m}}$ and all valuations $\nu \neq \nu_{\mathfrak{m}}$ for which $D_{\vec{v}} g \leq-n^{-1}$, where $\vec{v}$ is the tangent vector at $\nu$ represented by $\nu_{\mathfrak{m}}$. Then $\left(\mathcal{T}_{n}\right)_{n \geq 1}$ forms an increasing sequence of trees such that the closure of their union equals $\operatorname{supp} g$. Hence we are done if we can show that $\mathcal{T}_{n}$ is finite.

The key remark is that we may de-localize the integrability condition which defines a tree potential. Specifically, pick tangent vectors $\vec{v}_{1}, \ldots, \vec{v}_{k}$ at different valuations $\nu_{1}, \ldots, \nu_{k}$, such that $\vec{v}_{i}$ is represented neither by $\nu_{\mathfrak{m}}$, nor by $\nu_{j}$ for $j \neq i$. Then $\sum_{1}^{k} D_{\vec{v}_{i}} g \leq g\left(\nu_{\mathfrak{m}}\right) \leq 1$. This implies that $\mathcal{T}_{n}$ has at most $n$ ends.

We endow $\mathcal{P}$ with the weak topology: $g_{k} \rightarrow g$ iff $g_{k}(\nu) \rightarrow g(\nu)$ for any $\nu \in \mathcal{V}_{\mathrm{qm}}$.

Lemma 5.5. $\mathcal{P}$ is a closed convex cone in $\mathcal{V}_{\mathrm{qm}}^{\mathbf{R}}$ and $\mathcal{P}_{1}=\left\{g \in \mathcal{P} \mid g\left(\nu_{\mathfrak{m}}\right)=1\right\}$ is compact.

\footnotetext{
${ }^{6}$ Note that in $[\mathrm{FJ} 1], \mathcal{P}$ is denoted by $\mathcal{P}^{+}$, and that tree potentials are positive tree potentials.
} 
Proof. It is clear that if $g$ and $h$ are tree potentials, then so is $a g+b h$ for any $a, b>0$. Let us show that $\mathcal{P}$ is weakly closed. Condition (P1) is easily seen to be preserved under pointwise limits. The same is true for (P2)-(P3) by the following elementary fact: pick a sequence $f_{j}$ of concave functions converging pointwise towards $f$ on an open real interval. Denote by $f^{\prime}(x+)\left(f^{\prime}(x-)\right)$ the right (left) derivative of $f$ at $x$. Then $f^{\prime}(x+) \leq \liminf f_{j}^{\prime}(x+) \leq \limsup f_{j}^{\prime}(x-) \leq f^{\prime}(x-)$.

As $\mathcal{V}$ is compact, the set $\mathcal{F}$ of functions on $\mathcal{V}$ with values in $\overline{\mathbf{R}}_{+}$is compact. By (P1), any $g \in \mathcal{P}$ extends uniquely to an element of $\mathcal{F}$. The above argument shows that the closure of $\mathcal{P}$ in $\mathcal{F}$ is the union of $\mathcal{P}$ and the function identically $+\infty$. Hence $\mathcal{P}_{1}$ is closed in $\mathcal{F}$ and therefore weakly compact.

Lemma 5.6. Any tree potential on $\mathcal{V}$ is lower semicontinuous and restricts to a continuous tree potential on any finite subtree.

Proof. Condition (P1) implies that the restriction of $g$ to any finite subtree $\mathcal{T} \subset \mathcal{V}$ is continuous. Denote by $g_{\mathcal{T}}$ the function defined by $g(\nu)=g\left(\nu_{0}\right)$ where $\nu_{0}=$ $\max \left[\nu_{\mathfrak{m}}, \nu\right] \cap \mathcal{T}$. This is a tree potential on $\mathcal{V}$ which coincides with $g$ on $\mathcal{T}$ and is continuous on $\mathcal{V}$. By Lemma 5.4 the support of $g$ is contained in the closure of an increasing union of finite subtrees $\mathcal{T}_{n}$. Set $g_{n}=g_{\mathcal{T}_{n}}$. Then $g_{n}$ is continuous and increases pointwise to $g$ as $n \rightarrow \infty$. Thus $g$ is lower semicontinuous.

However, tree potentials are not necessarily continuous: see Example 5.16.

Lemma 5.7. If $\left(g_{i}\right)_{i \in I}$ is any family of tree potentials, then $g=\inf _{i} g_{i}$ is also a tree potential.

Proof. This is proved in the same way as the fact that the infimum of a family of concave functions on $\mathbf{R}$ is concave. The details are left to the reader.

5.3. The tree Laplacian. Let us show how Borel measures give rise to tree potentials. First pick any valuation $\mu \in \mathcal{V}$ and define $g_{\mu}: \mathcal{V}_{\mathrm{qm}} \rightarrow[1, \infty)$ by $g_{\mu}(\nu)=\nu \cdot \mu$, where $\nu \cdot \mu=\alpha(\nu \wedge \mu)$ is the intersection product.

Lemma 5.8. The function $g_{\mu}$ is a tree potential.

Proof. Clearly $g_{\mu}$ is nonnegative, increasing and concave along totally ordered segments, and $g_{\mu}\left(\nu_{\mathfrak{m}}\right)=1$. If $\vec{v} \in T \nu_{\mathfrak{m}}$, then $D_{\vec{v}} g_{\mu}=1$ if $\vec{v}$ is represented by $\mu$ and zero otherwise. If $\vec{v} \in T \nu, \nu \neq \mu$, then $D_{\vec{v}} g_{\mu}=-1$ if $\nu \geq \mu$ and $\vec{v}$ is represented by $\nu_{\mathfrak{m}}$, one if $\vec{v}$ is represented by $\mu$, and zero otherwise. This easily implies (P2)-(P3).

For an arbitrary positive measure $\rho \in \mathcal{M}$ we set

$$
g_{\rho}(\nu)=\int_{\mathcal{V}} g_{\mu}(\nu) d \rho(\mu)=\int_{\mathcal{V}} \mu \cdot \nu d \rho(\mu) .
$$

As $\mathcal{V}$ is compact, any Borel measure may be weakly approximated by a finite atomic measure (see e.g. [Bour]), hence $g_{\rho}$ is a tree potential in view of Lemma 5.5. We now describe the general identification of tree potentials with positive measures on $\mathcal{V}$. This identification is analogous to the identification of subharmonic functions (modulo harmonic functions) with positive measures on $\mathbf{R}^{n}$. 
Theorem 5.9. [FJ1, Theorems 7.61 and 7.64] The map

$$
\mathcal{M} \ni \rho \mapsto g_{\rho} \in \mathcal{P}
$$

is a homeomorphism in the weak topology.

We refer to [FJ1] for a proof of this result.

Definition 5.10. The Laplacian $\Delta g$ of a tree potential $g \in \mathcal{P}$ is by definition the unique positive Borel measure such that $g_{\Delta g}=g$.

Example 5.11. If $\phi \in R$ is irreducible, then $\nu(\phi)=m(\phi) \nu \cdot \nu_{\phi}$, hence $\nu \mapsto \nu(\phi)$ defines a tree potential whose Laplacian is $m(\phi) \nu_{\phi}$.

The following properties characterize the measure $\Delta g$ : see Remark 5.2.

Proposition 5.12. Let $g \in \mathcal{P}$. The measure $\rho=\Delta g$ is the unique positive measure on $\mathcal{V}$ such that $\rho U(\vec{v})=D_{\vec{v}} g$ for any tangent vector $\vec{v}$ not represented by $\nu_{\mathfrak{m}}$; and $\rho U(\vec{v})=g\left(\nu_{\mathfrak{m}}\right)+D_{\vec{v}} g$ when $\nu_{\mathfrak{m}}$ represents $\vec{v}$. In particular, the total mass of $\Delta g$ is given by $g\left(\nu_{\mathfrak{m}}\right)$. Moreover,

$$
\rho\left\{\nu_{\mathfrak{m}}\right\}=g_{\rho}\left(\nu_{\mathfrak{m}}\right)-\sum_{\vec{v} \in T \nu_{\mathfrak{m}}} D_{\vec{v}} g_{\rho} \quad \text { and } \quad \rho\{\nu\}=-\sum_{\vec{v} \in T \nu} D_{\vec{v}} g_{\rho} \text { for } \nu \neq \nu_{\mathfrak{m}} .
$$

The proof essentially follows by linearity from the case $\rho=\mu$, and is left to the reader. We shall also need:

Corollary 5.13. If $g \in \mathcal{P}$ and $\nu \in \mathcal{V}_{\mathrm{qm}}$, then $g(\nu) \leq g\left(\nu_{\mathfrak{m}}\right) \alpha(\nu)$, with equality iff $\Delta g$ is supported on $\{\mu \geq \nu\}$.

Proof. We may assume $g\left(\nu_{\mathfrak{m}}\right)=$ mass $\Delta g=1$. Then $g(\nu)=1+\int_{\nu_{\mathfrak{m}}}^{\nu} \Delta g\left\{\mu^{\prime} \geq\right.$ $\mu\} d \alpha(\mu) \leq 1+\alpha(\nu)-1$ and equality holds iff $\Delta g$ is supported on $\{\mu \geq \nu\}$.

Remark 5.14. The representation $g=\int g_{\mu} d \rho_{g}(\mu)$ gives the Choquet decomposition of the tree potential $g$ in the closed convex cone $\mathcal{P}$ : the extremal points of $\mathcal{M}$ are Dirac masses so the extremal points of $\mathcal{P}$ are of the form $g_{\mu}$.

Proposition 5.15. Let $\mathcal{P}^{\prime}$ be the smallest closed positive subcone of $\mathcal{V}_{\mathrm{qm}}^{\mathbf{R}}$ which is closed under infima and contains all functions of the form $\nu \mapsto \nu(\phi)$ for $\phi \in R$ irreducible. Then $\mathcal{P}^{\prime}=\mathcal{P}$.

Proof. By Proposition 5.5, Lemma 5.7 and Example 5.11 we have $\mathcal{P}^{\prime} \subset \mathcal{P}$. For the reverse inclusion it suffices to show that $g_{\mu} \in \mathcal{P}^{\prime}$ for $\mu \in \mathcal{V}$. As the set of divisorial valuations is dense in $\mathcal{V}$, we may assume $\mu$ is divisorial. But then we can find $\phi_{1}, \phi_{2} \in R$ irreducible such that $\mu=\nu_{\phi_{1}} \wedge \nu_{\phi_{2}}$. Hence $g_{\mu}(\nu)=\min \left\{\nu\left(\phi_{1}\right), \nu\left(\phi_{2}\right)\right\}$, so $g_{\mu} \in \mathcal{P}^{\prime}$.

Example 5.16. Let $\rho=\sum_{n \geq 1} n^{-2} \nu_{y+n x}$. If $\nu_{n}=\nu_{y+n x, n^{3}}$, then $\nu_{n} \rightarrow \nu_{\mathfrak{m}}$ but $g_{\rho}\left(\nu_{n}\right)>n \rightarrow \infty>g_{\rho}\left(\nu_{\mathfrak{m}}\right)$. This shows that tree potentials are not weakly continuous in general (see Lemma 5.6). 
5.4. Intersection of measures. Using bilinearity we extend the intersection product on valuations in $\mathcal{V}$ to measures in $\mathcal{M}$. More precisely, if $\rho, \sigma \in \mathcal{M}$ then we define

$$
\rho \cdot \sigma:=\iint_{\mathcal{V} \times \mathcal{V}} \mu \cdot \nu d \rho(\mu) d \sigma(\nu)=\int_{\mathcal{V}} g_{\rho}(\nu) d \sigma(\nu)=\int_{\mathcal{V}} g_{\sigma}(\mu) d \rho(\mu),
$$

where $g_{\sigma}$ and $g_{\rho}$ are the tree potentials of $\rho$ and $\sigma$, respectively. The last two equalities follow from Fubini and the definition of $g_{\rho}$ and $g_{\sigma}$.

Since $\mu \cdot \nu \geq 1$ for all $\mu, \nu$ we get

$$
\rho \cdot \sigma \geq \operatorname{mass} \rho \cdot \operatorname{mass} \sigma=g_{\rho}\left(\nu_{\mathfrak{m}}\right) g_{\sigma}\left(\nu_{\mathfrak{m}}\right)
$$

with equality iff the supports of $g_{\rho}$ and $g_{\sigma}$ intersect only at $\nu_{\mathfrak{m}}$.

Proposition 5.17. The intersection product is lower semicontinuous on $\mathcal{M}$.

We refer to [FJ1, Proposition 7.76] for a proof of this result. Note that the intersection product is not continuous on $\mathcal{M}$, and not even on $\mathcal{V}$ as exemplified by $\nu_{n}=\nu_{y-n x, 2}$ : here $\nu_{n} \cdot \nu_{n}=2$ but $\nu_{n} \rightarrow \nu_{\mathfrak{m}}$ and $\nu_{\mathfrak{m}} \cdot \nu_{\mathfrak{m}}=1$.

\section{TREe TRANSFORMS OF PSH FUNCTIONS}

Fix a psh function $u$ and consider the real-valued function $g_{u}$ on $\mathcal{V}_{\mathrm{qm}}$ given by $g_{u}(\nu)=\nu(u)$, where $\nu(u)$ is defined by Theorem 3.1. We call $g_{u}$ the tree transform of $u$. We will show that $g_{u}$ is a tree potential. Its Laplacian $\rho_{u}=\Delta g_{u}$ is a measure on $\mathcal{V}$ called the tree measure of $u$ and contains a lot of information on $u$. We will try to understand what measures on $\mathcal{V}$ arise in this way.

6.1. Tree transforms are tree potentials. Our main goal is to prove

Theorem 6.1. The tree transform $g_{u}$ of any psh function $u$ is a tree potential on $\mathcal{V}$ and the tree measure $\rho_{u}=\Delta g_{u}$ has mass equal to the Lelong number of $u$.

Moreover, $\rho_{u}$ puts no mass on formal (i.e. non-analytic) curve valuations and its mass on an analytic curve valuation $\nu_{D}$ is related to the mass of $d d^{c} u$ on $D$ as follows: $\rho_{u}\left\{\nu_{D}\right\} \geq \lambda m(D)$ iff $d d^{c} u \geq \lambda[D]$.

Let us again emphasize that $\rho_{u}$ gives a very fine measurement of the singularity of $u$ at 0 . We shall see in Section 8 , that $\rho_{u}$ determines essentially all generalized Lelong numbers of $u$ in the sense of Demailly. Here we prove

Proposition 6.2. For two psh functions $u$ and $v$, the following three assertions are equivalent.

(1) for all modifications $\pi: X \rightarrow\left(\mathbf{C}^{2}, 0\right)$, and all points $p \in \pi^{-1}\{0\}$, we have $\nu^{\mathrm{L}}\left(\pi^{*} u, p\right)=\nu^{\mathrm{L}}\left(\pi^{*} v, p\right)$

(2) $u$ and $v$ have the same tree transform: $g_{u}=g_{v}$;

(3) $u$ and $v$ have the same tree measure: $\rho_{u}=\rho_{v}$.

Remark 6.3. It follows from Theorem 6.1 that if $(x, y)$ are local coordinates, then the function $t \mapsto \nu_{y, t}(u)$ is concave for $t \geq 1$. Thus we recover the fact [Ki2] that the Kiselman number $\nu_{a, b}^{x, y}$ is a concave function of $(a, b)$.

Remark 6.4. If $u$ is psh with tree measure $\rho_{u} \in \mathcal{M}$, then for any $\nu \in \mathcal{V}_{\mathrm{qm}}$ quasimonomial we have $\nu(u)=\int_{\mathcal{V}} \nu \cdot \mu d \rho_{u}(\mu)$. This follows from (5.1). 
Remark 6.5. The tree transform $u \mapsto g_{u}$ inherits the main properties stated in Theorem 3.1: compatibility, monotonicity, homogeneity, tropicality and semicontinuity. In addition, if $u_{n}$ is the Demailly approximating sequence of $u$, it follows from Proposition 3.12 that $g_{u_{n}} \rightarrow g_{u}$ in $\mathcal{P}$, hence $\rho_{u_{n}} \rightarrow \rho_{u}$ in $\mathcal{M}$. These properties completely characterize the tree transform.

Example 6.6. The tree measure of $u=\log \max \{|x|,|y|\}$ is $\rho_{u}=\nu_{\mathfrak{m}}$, i.e. a Dirac mass at the multiplicity valuation $\nu_{\mathfrak{m}}$. More generally, if $\phi$ is an irreducible germ of multiplicity $m=m(\phi), x$ is a coordinate transverse to $x$ as in Section 3.1, and $1 \leq t \leq \infty$, then the tree measure of $u=\log \max \left\{|\phi|^{1 / m},|x|^{t}\right\}$ is a Dirac mass at the valuation $\nu_{\phi, t}$. This follows from compatibility, homogeneity and tropicality.

The proof of Theorem 6.1, given below, goes by reduction to the algebraic case. We define the tree transform $g_{I}$ of an ideal $I \subset R$ by $g_{I}(\nu)=\nu(I):=\min _{\phi \in I} \nu(\phi)$.

Proposition 6.7. The tree transform $g_{I}$ of any ideal $I \subset R$ is a tree potential. Its Laplacian $\rho_{I}=\Delta g_{I}$ has mass $m(I):=\nu_{\mathfrak{m}}(I)$ and is an atomic measure supported on finitely many divisorial and (analytic) curve valuations.

Remark 6.8. In [FJ1, Theorem 8.2] we characterize measures on $\mathcal{V}$ of the form $\rho_{I}$ : they are atomic measures whose mass is a multiple of the generic multiplicity at any divisorial valuation, and a multiple of the multiplicity at any curve valuation.

Proof. For $\phi \in \mathfrak{m}$, set $g_{\phi}(\nu)=\nu(\phi)$. When $\phi$ is irreducible, Example 5.11 shows that $g_{\phi}$ is a tree potential and that $g_{\phi}$ is a piecewise affine function with integer slopes on any segment in $\mathcal{V}$ parameterized by skewness. By additivity in $\mathcal{P}$ and unique factorization in $R$, the same properties hold when $\phi$ is reducible.

Let $S \subset I$ be a finite set of generators for $I$. Then $g_{I}=\min _{\phi \in S} g_{\phi}$. Thanks to Lemma 5.7, $g_{I}$ is a tree potential. By Proposition 5.12, the mass of $\rho_{I}:=\Delta g_{I}$ is given by $g_{I}\left(\nu_{\mathfrak{m}}\right)=m(I)$.

It is clear that $g_{I}$ is supported on the smallest subtree of $\mathcal{V}$ containing $\nu_{\mathfrak{m}}$ and any $\nu_{\psi}$, where $\psi$ ranges over the irreducible factors of the elements of $S$. This is a finite subtree $\mathcal{S}$. Moreover, it follows from the preceding computation that on any segment in $\mathcal{S}$ parameterized by skewness, $g_{I}$ is a piecewise affine function with integer slopes. Thus $\rho_{I}$ is a finite sum of point masses, taken over valuations that are either ends or branch points in $\mathcal{S}$, or regular points in $\mathcal{S}$ where $g_{I}$ fails to be locally affine. From the integer slope property we conclude that $\rho_{I}=\sum_{i=1}^{r} n_{i} \nu_{i}$, where $\nu_{i}$ are divisorial (i.e. have rational skewness) or curve valuations and $n_{i}$ are positive integers.

Proof of Theorem 6.1. First suppose $u$ has logarithmic singularities, and write $u=$ $\frac{c}{2} \log \sum_{i=1}^{n}\left|\phi_{i}\right|^{2}$ for holomorphic $\phi_{i}$ and $c>0$. Then $g_{u}(\nu)=c g_{I}(\nu)=c \min \nu\left(\phi_{i}\right)$, where $I$ is the ideal generated by the $\phi_{i}$ (see Proposition 3.11). Hence $g_{u}$ is a tree potential in this case by Proposition 6.7. Further, the mass of $\Delta g_{u}$ equals $m(I)$, which is the Lelong number of $u$.

In the general case, we use Proposition 3.12. Let $u_{n}$ be a sequence of psh functions with logarithmic singularities such that $u_{n} \rightarrow u$, and $g_{u_{n}} \rightarrow g_{u}$ pointwise. Since $\mathcal{P}$ is weakly closed (Lemma 5.5) it follows that $g_{u}$ is a tree potential. That $\Delta g_{u}$ has the right mass follows since $g_{u_{n}}\left(\nu_{\mathfrak{m}}\right) \rightarrow g_{u}\left(\nu_{\mathfrak{m}}\right)$. 
For the second assertion, first consider an irreducible holomorphic germ $\phi \in \mathfrak{m}$, and suppose $d d^{c} u \geq \lambda[D]$, where $D=\{\phi=0\}$ and $\lambda>0$. Then $u \leq \lambda \log |\phi|$ and $g_{u}\left(\nu_{\phi, t}\right) \geq \lambda m(\phi) t$ for all $t \geq 1$. This implies $\rho_{u}\left\{\nu_{\phi}\right\} \geq \lambda m(\phi)$.

Conversely, suppose $\phi \in \hat{\mathfrak{m}}$ is a formal germ and $\rho_{u}\left\{\nu_{\phi}\right\} \geq \lambda m(\phi)$ with $\lambda>0$. Fix any $\varepsilon>0$. Then $g_{u}\left(\nu_{\phi, t}\right) \geq(\lambda-\varepsilon) m(\phi) t$ for large $t$.

First assume $u=\frac{c}{2} \log \sum_{i=1}^{n}\left|\phi_{i}\right|^{2}$ has logarithmic singularities. Then $g_{\phi_{i}}\left(\nu_{\phi, t}\right) \geq$ $c^{-1}(\lambda-\varepsilon) m(\phi) t$ for all $i$, which implies that $\phi^{k}$ divides $\phi_{i}$ for all $i$, where $k$ is the smallest integer larger than $c^{-1} \lambda$. This implies that the curve $D=\{\phi=0\}$ is analytic, and that $u \leq c k \log |\phi|+O(1)$. Hence $d d^{c} u \geq c k[D] \geq \lambda[D]$.

In the general case we have $\left|g_{u_{n}}\left(\nu_{\phi, t}\right)-g_{u}\left(\nu_{\phi, t}\right)\right| \leq A\left(\nu_{\phi, t}\right) / n \leq m(\phi) t / n$ by (3.9) and the estimates from Section 1.8. Hence, for $n$ large, $g_{u_{n}}\left(\nu_{\phi, t}\right) \geq(\lambda-2 \varepsilon) m(\phi) t$ for large $t$. By what precedes $D=\{\phi=0\}$ is analytic, $d d^{c} u_{n}$ puts mass at least $\lambda$ on $D$, and $u_{n} \leq \lambda \log |\phi|+O(1)$. By (2.2), $u \leq \lambda \log |\phi|+O(1)$, so $d d^{c} u \geq \lambda[D]$.

Proof of Proposition 6.2. The equivalence of (2) and (3) is a consequence of Theorem 5.9. The implication $(2) \Rightarrow(1)$ follows from Proposition 4.1, and the following fact. The Lelong number at a point $p$ equals the Lelong number at a generic point on the exceptional divisor obtained by blowing up $p$. Finally, suppose (1) is true. Then Proposition 4.1 shows that $g_{u}(\nu)=g_{v}(\nu)$ for all divisorial valuations $\nu$. As tree potentials are continuous on finite subtrees by Lemma 5.6, and divisorial valuations are dense on any finite subtree in $\mathcal{V}_{\mathrm{qm}}$, we conclude $g_{u}=g_{v}$.

6.2. Representation of measures by psh functions. When $\rho=\rho_{u} \in \mathcal{M}$ is the tree measure of a psh function $u$, we say that $\rho$ is represented by $u$. In general it seems quite hard to characterize the measures in $\mathcal{M}$ that are represented by psh functions (or currents). On the one hand, Example 6.6 shows that any Dirac mass at a quasimonomial or analytic curve valuation is represented by a psh function. By taking sums and limits we obtain many more measures: see Example 7.4 for an interesting example.

On the other hand, there are also some restrictions. The tree measure of a psh function cannot put mass on any formal curve valuation by Theorem 6.1. There are likely some restrictions at infinitely singular valuations, too, but we do have

Proposition 6.9. Let $\nu$ be an infinitely singular valuation, given by a Puiseux series $\hat{\phi}=\sum_{1}^{\infty} a_{j} x^{\hat{\beta}_{j}}$ as in Section 1.9. Suppose there exists $r>0$ such that $\sum_{1}^{\infty}\left|a_{j}\right|^{2} \hat{\beta}_{j} r^{2 \hat{\beta}_{j}}<\infty$. Then $\nu$ is represented by a psh function.

With stronger assumptions, one should be able to analyze more precisely the set of psh functions (or currents) representing $\nu$. One may for instance ask for conditions on the $a_{j}$ 's ensuring the existence of a unique extremal positive closed $(1,1)$-current representing $\nu$. We refer to [St] for related problems and to $[\mathrm{Kw}$, Section 6] for results in this direction.

Proof. We may assume that $(x, y)$ in the definition of the Puiseux series are global coordinates on $\mathbf{C}^{2}$. Let $\hat{\phi}_{n}=\sum_{1}^{n} a_{j} x^{\hat{\beta}_{j}}$ as above, and let $\phi_{n} \in R$ be the minimal polynomial of $\hat{\phi}_{n}$. Write $m_{n}=m\left(\phi_{n}\right)$. The divisorial valuation $\nu_{k}=\nu_{\phi_{n}} \wedge \nu_{\phi_{k}}$ is independent of $n$ for $n>k$ and $\nu_{k}$ increases to $\nu$ as $k \rightarrow \infty$. 
Pick $r_{0}>0$ such that $\sum_{1}^{\infty}\left|a_{j}\right|^{2} \hat{\beta}_{j} r^{2 \hat{\beta}_{j}}<\infty$, and define $C=\sum_{1}^{\infty}\left|a_{j}\right|^{2} \hat{\beta}_{j} r_{0}^{2\left(\hat{\beta}_{j}-\hat{\beta}_{1}\right)}$. Let $B_{r}=\Delta_{r} \times \Delta_{1}$ for $r \in\left(0, r_{0}\right]$. Define $u_{n}=\frac{1}{m_{n}} \log \left|\phi_{n}\right|$ and $T_{n}=d d^{c} u_{n}$. Then $T_{n}$ forms a sequence of positive closed currents on $B_{r_{0}}$. Further, $T_{n}$ is the pushforward of the current of integration on the disk $\Delta_{n}=\left\{|t|<r_{0}^{1 / m_{n}}\right\}$ by the $\operatorname{map} \psi_{n}(t)=\left(t^{m_{n}}, \sum_{1}^{n} a_{j} t^{m_{n} \hat{\beta}_{j}}\right)$. Notice that $\psi_{n}\left(\Delta_{n}\right) \in\left\{|x|<r_{0},|y| \leq C|x|^{\hat{\beta}_{1}}\right\}$.

Let $b_{n}(r)$ be the mass of (the trace measure of) $T_{n}$ in $B_{r}$. We have $b_{n}(r)=$ $\pi\left(r^{2}+\sum\left|a_{j}\right|^{2} \hat{\beta}_{j} r^{2 \hat{\beta}_{j}}\right)$, hence $b_{n}(r) \leq \pi\left(r^{2}+C r^{2 \hat{\beta}_{1}}\right)$ for $r<r_{0}$. Thus we may extract a subsequence $T_{n_{j}}$ that converges to a positive closed current $T$ in $B_{r_{0}}$. The mass $b(r)$ of $T$ in $B_{r}$ also satisfies $b(r) \leq \pi\left(r^{2}+C r^{2 \hat{\beta}_{1}}\right)$. As $\hat{\beta}_{1}>1$, this implies that the Lelong number of $T$ is at most 1 (see 2.6).

On the other hand, for $n \geq k$ we have $\nu_{k}\left(T_{n}\right)=\nu_{k}\left(\phi_{n}\right) / m_{n}=\alpha\left(\nu_{k}\right)$. By semicontinuity this gives $\nu_{k}(T) \geq \alpha\left(\nu_{k}\right) \geq \alpha\left(\nu_{k}\right) \nu_{\mathfrak{m}}(T)$. Let $\rho=\rho_{T}$ be the measure represented by $T$. By Corollary 5.13, $\rho$ has mass 1 and is supported on $\left\{\mu \geq \nu_{k}\right\}$ for all $k$. Thus $\rho$ is a point mass at $\nu$, completing the proof.

\section{Attenuation of singularities of Currents}

Positive closed $(1,1)$-currents in many ways generalize curves (in dimension 2). Here we shall prove a theorem that generalizes embedded resolution of plane curve singularities.

Theorem 7.1. Let $T$ be any positive closed $(1,1)$-current near the origin in $\mathbf{C}^{2}$. Then for any $\eta, \varepsilon>0$ there exists a modification $\pi: X \rightarrow\left(\mathbf{C}^{2}, 0\right)$ and positive closed $(1,1)$-currents $S_{1}, S_{2}$ on $X$, such that $\pi^{*} T=S_{1}+S_{2}$ and:

- the support of $S_{1}$ is a curve with normal crossing singularities;

- $\sup _{p \in \pi^{-1}(0)} \nu^{\mathrm{L}}\left(S_{2}, p\right)^{1+\eta} \leq \sum_{p \in \pi^{-1}(0)} \nu^{\mathrm{L}}\left(S_{2}, p\right)^{1+\eta} \leq \varepsilon$.

This statement implies the corresponding global statement, when $T$ is defined on a compact complex surface. Thus Theorem 7.1 strengthens the main result of $[\mathrm{G}]$ in two ways, as the method there - borrowed from [Mi] - only gives the weaker bound $\sum_{\pi^{-1}(0)} \nu^{\mathrm{L}}\left(S_{2}, p\right)^{2} \leq \varepsilon$. We also recover the following result by Mimouni [Mi, Théorème III.1.2]. See below for a definition of strict transform.

Corollary 7.2. Suppose $T$ does not charge any curve, and fix $\varepsilon>0$. Then there exists a modification $\pi: X \rightarrow\left(\mathbf{C}^{2}, 0\right)$ such that the strict transform of $T$ by $\pi$ has all its Lelong numbers bounded by $\varepsilon$.

Remark 7.3. Theorem 7.1 fails for $\eta=0$ in general. As the proof of Lemma 7.6 below shows, any psh function $T$ whose tree measure $\rho_{T}$ has no atoms and is supported on the set of smooth, analytic, curve valuations, yields a counterexample. See Remark 7.8 for more details and Example 7.4 for an explicit construction.

Example 7.4. Let $\Sigma:=\{-1,+1\}^{\mathbf{N}^{*}}$, and $\rho$ be the uniformly distributed measure on $\Sigma$, i.e. the product measure $\rho=\otimes_{1}^{\infty} \rho_{i}$, where $\rho_{i}\{+1\}=\rho_{i}\{-1\}=1 / 2$ for any $i$. For $\sigma=\left(\sigma_{i}\right)_{1}^{\infty} \in \Sigma$ set $f_{\sigma}(x):=\sum_{i \geq 1} \sigma_{i} x^{i}$. Now define

$$
T=\int_{\Sigma}\left[y=f_{\sigma}(x)\right] d \rho(\sigma)
$$


where $\left[y=f_{\sigma}(x)\right]$ denotes the current of integration on the curve $\left\{y=f_{\sigma}(x)\right\}$. The set $\Sigma$ can be thought of as a Cantor set on two symbols, and the collection $\left\{y=f_{\sigma}(x)\right\}_{\sigma \in \Sigma}$, as a "Cantor bouquet" of smooth curves. Indeed, by sending $\sigma \in \Sigma$ to the curve valuation $\nu_{y-f_{\sigma}(x)}$ we obtain a homeomorphism of $\Sigma$ (with the product topology) onto a compact subset of $\mathcal{V}$. The tree measure $\rho_{T}$ of $T$ on $\mathcal{V}$ is then the pushforward of $\rho$ on $\Sigma$.

Under any modification $\pi$, the strict transform of the bouquet will be a finite union of bouquets all of which are isomorphic to the original one. See Figure 5. The sum of the Lelong numbers of the strict transform of $T$ will always equal one, the Lelong number of $T$ at the origin.
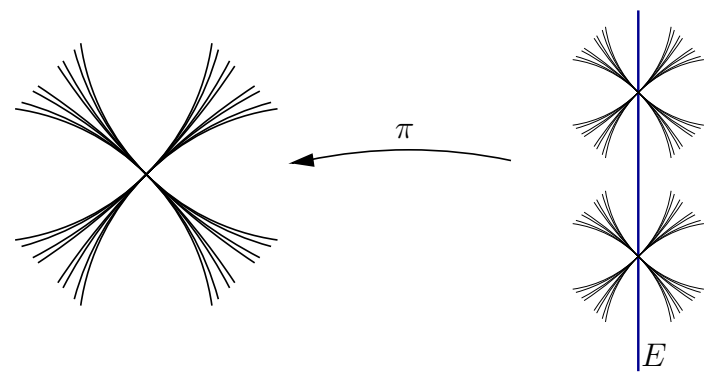

Figure 5. The Cantor bouquet of curves splits into two isomorphic parts after a single blowup of the origin. See Example 7.4.

Remark 7.5. Let us note a strong similarity between the statement of Theorem 7.1 and the definition of Zariski's decomposition as in [BDPP, Theorem 3.1] (see also [Bouc]).

Proof of Theorem 7.1. We shall prove that we may pick $\pi$ such that $\pi^{*} T=S_{1}+$ $S_{2}$ as in the statement of the theorem with $\sup _{p} \nu^{\mathrm{L}}\left(S_{2}, p\right)$ arbitrarily small and $\sum_{p} \nu^{\mathrm{L}}\left(S_{2}, p\right)$ uniformly bounded. This will make the $\ell^{1+\eta}$-norm arbitrarily small.

By Siu's Theorem, $T=T_{1}+T_{2}$, where $T_{1}=\sum_{j=1}^{n} a_{j}\left[D_{j}\right]$ with $a_{j}>0$ and $D_{j}$ irreducible, and $T_{2} \nsupseteq \varepsilon[D]$ for all irreducible curves $D$.

Denote by $\Gamma_{\pi}^{*}$ the set of exceptional components of a modification $\pi$. The pullback $\pi^{*} T$ is a positive closed $(1,1)$-current which charges any curve $E \in \Gamma_{\pi}^{*}$; more precisely $\pi^{*} T \geq \operatorname{div}_{E}\left(\pi^{*} T\right)[E]$ for any $E \in \Gamma_{\pi}^{*}$. By Proposition 4.1, $\operatorname{div}_{E}\left(\pi^{*} T\right)$ is also the Lelong number of $\pi^{*} T$ at a generic point $p \in E$. The strict transform of $T$ by $\pi$ is by definition

$$
T^{\prime}:=\pi^{*} T-\sum_{E \in \Gamma_{\pi}^{*}} \operatorname{div}_{E}\left(\pi^{*} T\right)[E] .
$$

It is a positive closed $(1,1)$-current with zero Lelong number except on a countable subset of $\pi^{-1}(0)$.

We now prove that we may pick $\pi$ such that the Lelong numbers of the strict transform of $T_{2}$ are arbitrarily small. As we shall see, composing $\pi$ with further blowups does not destroy the latter property. Hence we may assume that the total 
transform of the curve $\bigcup D_{j}$ has simple normal crossings, so that the theorem holds with $S_{2}$ being the strict transform of $T_{2}$ and $S_{1}=\pi^{*} T-S_{2}$.

By this argument, we are reduced to the case $T_{1}=0$. In other words, we may assume that $T \nsupseteq \varepsilon[D]$ for all irreducible curves $D$. We shall pick $S_{2}=T^{\prime}$, the strict transform of $T$.

Let $\rho_{T}$ be the tree measure of $T$. The assumption on $T$ means that $\rho_{T}\left\{\nu_{D}\right\}<$ $\varepsilon m(D)$ for all $D$ : see Theorem 6.1. Our aim is to estimate the Lelong numbers of $T^{\prime}$ in terms of the mass of $\rho_{T}$ on particular subregions in the valuative tree. To do so, we rely in an essential way on the results described in Section 1.10.

Consider any modification $\pi: X \rightarrow\left(\mathbf{C}^{2}, 0\right)$ and pick a point $p \in \pi^{-1}(0)$. Denote by $\nu_{p}$ the divisorial valuation associated to the blowup of $p$, and define an open subset $U(p) \subset \mathcal{V}$ as follows (see Figure 3 on p.9 and compare with Proposition 1.1):

- If $p$ is a regular point on $\pi^{-1}(0)$, lying on a unique exceptional component $E$, then $\nu_{p}>\nu_{E}$ and $U(p):=\left\{\mu ; \mu \wedge \nu_{p}>\nu_{E}\right\}$.

- If $p$ is a singular point on $\pi^{-1}(0)$, then $p=E \cap E^{\prime}$, where $\nu_{E}<\nu_{p}<\nu_{E^{\prime}}$, and $U(p):=\left\{\mu ; \nu_{E}<\mu \wedge \nu_{E^{\prime}}<\nu_{E^{\prime}}\right\}$.

In can be shown that $U(p)$ is exactly the set of valuations in $\mathcal{V}$ whose center on $X$ is the point $p$ : see [FJ1, Proposition 6.32]. For fixed $\pi$, the sets $U(p)$ form a disjoint open cover of $\mathcal{V} \backslash\left\{\nu_{E} ; E \in \Gamma_{\pi}^{*}\right\}$. Recall that $\nu^{\mathrm{L}}\left(T^{\prime}, p\right)$ denotes the Lelong number of the current $T^{\prime}$ at $p$. We shall prove:

Lemma 7.6. For any current $T$, any modification $\pi: X \rightarrow\left(\mathbf{C}^{2}, 0\right)$ and any point $p \in \pi^{-1}(0)$ we have $\rho_{T}(U(p)) \geq b\left(\nu_{p}\right) \nu^{\mathrm{L}}\left(T^{\prime}, p\right)$.

Lemma 7.7. Let $\rho \in \mathcal{M}$ be a positive measure on $\mathcal{V}$ such that $\rho\left\{\nu_{D}\right\}<\varepsilon m(D)$ for every irreducible curve $D$. Then there exists a modification $\pi: X \rightarrow\left(\mathbf{C}^{2}, 0\right)$ such that $\rho(U(p)) \leq \varepsilon b\left(\nu_{p}\right)$ for every $p \in \pi^{-1}(0)$.

In view of the reductions above, we obtain, for any $\varepsilon>0$, the existence of $\pi$ such that $\nu^{\mathrm{L}}\left(T^{\prime}, p\right) \leq \varepsilon$ for all $p \in \pi^{-1}(0)$. On the other hand, since $b\left(\nu_{p}\right) \geq 1$ for all $p$ and the sets $U(p)$ are disjoint, Lemma 7.6 shows that $\sum_{p \in \pi^{-1}(0)} \nu^{\mathrm{L}}\left(T^{\prime}, p\right)$ is uniformly bounded by the mass of $\rho_{T}$, i.e. the Lelong number $\nu^{\mathrm{L}}(T)$ of $T$ at the origin. For $\eta>0$, we get $\sum_{p \in \pi^{-1}(0)} \nu^{\mathrm{L}}\left(T^{\prime}, p\right)^{1+\eta} \leq \varepsilon^{\eta} \nu^{\mathrm{L}}(T)$. If $\eta$ and $T$ are fixed, we can then make $\varepsilon^{\eta} \nu^{\mathrm{L}}(T)$ arbitrarily small. This concludes the proof of Theorem 7.1.

Proof of Lemma 7.6. To simplify notation, we shall write $b_{E}$ for $b\left(\nu_{E}\right), \alpha_{E}$ for $\alpha\left(\nu_{E}\right)$ etc. Let $\mu_{p}$ be the multiplicity valuation at $p$ in $X$ and write $U=U(p)$.

First suppose $p \in E$ is a regular point of $\pi^{-1}(0)$. By definition $T^{\prime}=\pi^{*} T-$ $\operatorname{div}_{E}\left(\pi^{*} T\right)[E]$. In particular $\nu^{\mathrm{L}}\left(T^{\prime}, p\right)=\mu_{p}\left(\pi^{*} T\right)-\operatorname{div}_{E}\left(\pi^{*} T\right)$. We have $\nu_{E}=$ $b_{E}^{-1} \pi_{*} \operatorname{div}_{E}$ and $\nu_{p}=b_{p}^{-1} \pi_{*} \mu_{p}$. Moreover, $b_{p}=b_{E}$ by Proposition 1.1 (i), so

$$
\nu^{\mathrm{L}}\left(T^{\prime}, p\right)=b_{p}\left(\nu_{p}(T)-\nu_{E}(T)\right) .
$$

By Lemma 1.2 we have $\alpha_{p}-\alpha_{E}=b_{p}^{-2}$. Using Remark 6.4 we have

$$
\begin{aligned}
\nu_{p}(T)-\nu_{E}(T) & =\int_{\mathcal{V}}\left(\nu_{p} \cdot \nu-\nu_{E} \cdot \nu\right) d \rho_{T}(\nu)=\int_{U}\left(\nu_{p} \cdot \nu-\nu_{E} \cdot \nu\right) d \rho_{T}(\nu) \\
& \leq\left(\alpha_{p}-\alpha_{E}\right) \rho_{T}(U)=b_{p}^{-2} \rho_{T}(U) .
\end{aligned}
$$


This concludes the proof in this case.

Now suppose $p=E \cap E^{\prime}$ is a singular point of $\pi^{-1}(0)$. Then $T^{\prime}=\pi^{*} T-$ $\operatorname{div}_{E}\left(\pi^{*} T\right)[E]-\operatorname{div}_{E^{\prime}}\left(\pi^{*} T\right)\left[E^{\prime}\right]$. We have $\nu_{E}=b_{E}^{-1} \pi_{*} \operatorname{div}_{E}, \nu_{E^{\prime}}=b_{E^{\prime}}^{-1} \pi_{*} \operatorname{div}_{E^{\prime}}$ and $\nu_{p}=b_{p}^{-1} \pi_{*} \nu_{p}$. Moreover, $b_{p}=b_{E}+b_{E^{\prime}}$ by Proposition 1.1 (ii), so

$$
\nu^{\mathrm{L}}\left(T^{\prime}, p\right)=\left(b_{E}+b_{E^{\prime}}\right) \nu_{p}(T)-b_{E} \nu_{E}(T)-b_{E^{\prime}} \nu_{E^{\prime}}(T) .
$$

We may assume that $\nu_{E}<\nu_{p}<\nu_{E^{\prime}}$. By Lemma 1.2 we then have $\alpha_{p}-\alpha_{E}=$ $b_{E}^{-1}\left(b_{E}+b_{E^{\prime}}\right)^{-1}$ and $\alpha_{E^{\prime}}-\alpha_{p}=b_{E^{\prime}}^{-1}\left(b_{E}+b_{E^{\prime}}\right)^{-1}$. This implies that

$$
\nu^{\mathrm{L}}\left(T^{\prime}, p\right)=\frac{1}{b_{p}}\left(\frac{\nu_{p}(T)-\nu_{E}(T)}{\alpha_{p}-\alpha_{E}}-\frac{\nu_{E^{\prime}}(T)-\nu_{p}(T)}{\alpha_{E^{\prime}}-\alpha_{p}}\right) .
$$

As above we have $\nu_{p}(T)-\nu_{E}(T) \leq\left(\alpha_{p}-\alpha_{E}\right) \rho_{T}\left\{\mu \wedge \nu_{p}>\nu_{E}\right\}$. Furthermore,

$$
\begin{aligned}
\nu_{E^{\prime}}(T)-\nu_{p}(T)= & \int_{\mathcal{V}}\left(\nu_{p} \cdot \nu-\nu_{E} \cdot \nu\right) d \rho_{T}(\nu) \\
& \geq \int_{\nu \geq \nu_{E^{\prime}}}\left(\nu_{p} \cdot \nu-\nu_{E} \cdot \nu\right) d \rho_{T}(\nu) \geq \rho_{T}\left\{\mu \geq \nu_{E^{\prime}}\right\}\left(\alpha_{E^{\prime}}-\alpha_{p}\right),
\end{aligned}
$$

where the first equality follows from Remark 6.4. Thus we conclude

$$
\nu^{\mathrm{L}}\left(T^{\prime}, p\right) \leq \frac{1}{b_{p}}\left(\rho_{T}\left\{\mu \wedge \nu_{F}>\nu_{E}\right\}-\rho_{T}\left\{\mu \geq \nu_{E^{\prime}}\right\}\right)=\frac{1}{b_{p}} \rho_{T}(U)
$$

which completes the proof of Lemma 7.6.

Remark 7.8. The proof shows that equality holds, i.e. $\rho_{T}(U(p))=b\left(\nu_{p}\right) \nu^{\mathrm{L}}\left(T^{\prime}, p\right)$, when $\rho_{T}$ is supported on the set of smooth curve valuation. Compare Remark 7.3.

Proof of Lemma 7.7. Define $\mathcal{T}=\{\nu \mid \rho\{\mu \geq \nu\} \geq \varepsilon m(\nu)\}$. It is clear that $\nu \in \mathcal{T}$, $\nu^{\prime} \leq \nu$ implies $\nu^{\prime} \in \mathcal{T}$, hence $\mathcal{T}$ is a subtree of $\mathcal{V}$. It is moreover a finite subtree, with at most $\varepsilon^{-1} \times$ mass $\rho$ ends. Our assumption implies that all these ends are quasimonomial valuations.

Now pick a modification $\pi: X \rightarrow\left(\mathbf{C}^{2}, 0\right)$ with the following properties:

(i) every end in $\mathcal{T}$ is dominated by $\nu_{E}$, for some exceptional component $E$;

(ii) whenever $E, E^{\prime}$ are exceptional components of $\pi$ that intersect in $X$, we have $b(E)+b\left(E^{\prime}\right)>\operatorname{mass} \rho / \varepsilon$.

We may achieve (i) as each end in $\mathcal{T}$ are quasimonomial, hence dominated by some divisorial valuation, which we may assume to be of the form $\nu_{E}$ for some exceptional component $E$ of $\pi$. If (ii) would fail for some pair $E, E^{\prime}$, then we may compose $\pi$ with the blowup at $E \cap E^{\prime}$. This creates a new exceptional component $F$ with $b(F)>\max \left\{b(E), b\left(E^{\prime}\right)\right\}$. Thus (ii) holds after finitely many further blowups.

Now pick any $p \in \pi^{-1}(0)$. If $p=E \cap E^{\prime}$ is a singular point on $\pi^{-1}(0)$, then the conclusion of Lemma 7.7 is immediate as $b(F)=b(E)+b\left(E^{\prime}\right)>\operatorname{mass} \rho / \varepsilon$.

Hence suppose $p$ is a regular point on $\pi^{-1}(0)$, belonging to a unique exceptional component $E$. Then $\nu_{p}$ does not represent the same tangent vector as any $\nu_{E}^{\prime}, E^{\prime}$ ranging over exceptional components of $\pi$. By (i) this implies that $U(p) \cap \mathcal{T}=\emptyset$. For any $\left.\nu \in] \nu_{E}, \nu_{p}\right]$ we thus have $\rho\{\mu \geq \nu\}<\varepsilon m(\nu)=\varepsilon b\left(\nu_{p}\right)$. As $\nu \rightarrow \nu_{E}$ we conclude $\rho(U(p)) \leq \varepsilon b\left(\nu_{p}\right)$, which completes the proof. 


\section{INTERSECTION FORMULA}

Our aim is to relate the mass at the origin of the intersection product of two positive closed $(1,1)$ currents to their tree measures. An optimistic guess is

$$
d d^{c} u \wedge d d^{c} v\{0\}=\rho_{u} \cdot \rho_{v}=\iint_{\mathcal{V} \times \mathcal{V}} \mu \cdot \nu d \rho_{u}(\mu) d \rho_{v}(\nu),
$$

where $\rho_{u}, \rho_{v}$ are the tree measures of $u$ and $v$, respectively. However, this is indeed too much to hope for.

Example 8.1. Let $u=\max \{-\sqrt{-\log |x|}, \log |y|\}$, and $v=\log |x|$. Then $d d^{c} u \wedge$ $d d^{c} v\{0\}$ equals the mass of $\left.d d^{c} u\right|_{x=0}$ which is one. On the other hand, the Lelong number of $u$ is zero, hence $\rho_{u}=0$ so $\rho_{u} \cdot \rho_{v}=0$ and (8.1) fails.

We may more precisely conjecture (8.1) as soon as neither current charges an analytic curve. Note that this would in particular imply that the admissible wedge product of two currents with zero Lelong number never charges the origin - something that seems quite hard to prove.

Our aim in this section is to give partial results in the direction of (8.1). We prove that equality holds when either $u$ or $v$ has logarithmic singularities (Proposition 8.2). We prove that a lower bound always holds (Proposition 8.5). Finally we show that equality holds whenever $v$ is a psh weight for which $e^{v}$ is Hölder continuous (Proposition 8.7). This gives an interpretation of a generalized Lelong number as an average of valuations.

\subsection{Psh functions with logarithmic singularities. Here we prove}

Proposition 8.2. Let $u$ and $v$ be psh functions such that $d d^{c} u \wedge d d^{c} v$ is admissible. Suppose we are in one of the following two cases:

- either $u$ is an arbitrary psh function, $v$ has logarithmic singularities and $d d^{c} v$ does not charge any curve;

- or $u, v$ both have logarithmic singularities.

Then (8.1) holds.

Remark 8.3. An algebraic version of this result can be found in [FJ1, Section 8.1.4]. Pick $I, J$ two analytic ideals, generated by finitely many $f_{i}$ 's and $g_{j}$ 's respectively and define $u=\frac{1}{2} \log \sum\left|f_{i}\right|^{2}, v=\frac{1}{2} \log \sum\left|g_{j}\right|^{2}$. Then the mass $d d^{c} u \wedge d d^{c} v\{0\}$ can be naturally interpreted as a mixed multiplicity of the ideals $I$ and $J$. Theorem 8.13 from [FJ1] asserts that in a quite general algebraic setting, the mixed multiplicity of two ideals can be computed using their tree transforms.

Proof of Proposition 8.2. First assume $v$ has logarithmic singularities and does not charge any curves. The tree transform of $v$ is a sum of Dirac masses at finitely many divisorial valuations, $\rho_{u}=\sum_{1}^{k} c_{i} \nu_{i}$.

Lemma 8.4. Let $v, v^{\prime}$ be two psh functions with logarithmic singularities, whose tree transforms coincide. Then the difference $v-v^{\prime}$ is a bounded function.

We hence choose for each $1 \leq i \leq k$, an irreducible $\phi_{i} \in \mathfrak{m}$, and $t_{i} \geq 1$ so that $\nu_{i}=\nu_{\phi_{i}, t_{i}}$. By the preceding lemma, and Proposition 2.2 we may replace $v$ by the $\operatorname{sum} \sum_{i=1}^{k} c_{i} \log \max \left\{\|p\|^{t_{i}},\left|\phi_{i}\right|^{1 / m_{i}}\right\}, m_{i}=m\left(\phi_{i}\right)$. By linearity of both sides of 
the equation (8.1), we are reduced to the case where the measure $\rho_{v}$ is supported at a single valuation, i.e. we can suppose $k=1$, and $v=\log \max \left\{\|p\|^{t},|\phi|^{1 / m}\right\}$, for an irreducible $\phi \in \mathfrak{m}$, and $t \geq 1$. Now $d d^{c} u \wedge d d^{c} v\{0\}=\nu_{\phi, t}(u)$ follows from Proposition 3.9. This concludes the proof in this case.

When $u$ and $v$ both have logarithmic singularities, we write $u=u^{\prime}+\sum a_{i} \log \left|\phi_{i}\right|$, $v=v^{\prime}+\sum b_{j} \log \left|\psi_{j}\right|$, where $d d^{c} u^{\prime}$ and $d d^{c} v^{\prime}$ do not charge any curve, $\phi_{i}, \psi_{j} \in \mathfrak{m}$ are irreducible analytic functions and $a_{i}, b_{j}>0$. By linearity and from what precedes we are reduced to the case $u=\log |\phi|, v=\log |\psi|$ for distinct irreducible $\phi, \psi \in \mathfrak{m}$. In this case, $d d^{c} u \wedge d d^{c} v$ is the pull-back under the finite map $F(z, w)=$ $(\phi(z, w), \psi(z, w))$ of the measure $\mu=d d^{c} \log |z| \wedge d d^{c} \log |w|$. The mass of $\mu$ at 0 is one, and the topological degree of $F$ is exactly the intersection product of the two curves $C=\{\phi=0\}$ and $D=\{\psi=0\}$. It follows that $d d^{c} u \wedge d d^{c} v\{0\}=C \cdot D$. The tree measure of $u$ (resp. $v$ ) is supported on $\nu_{C}$ (resp. $\nu_{D}$ ) and has mass $m(C)$ (resp. $m(D)$ ). Whence $\rho_{u} \cdot \rho_{v}=m(C) m(D) \nu_{C} \cdot \nu_{D}=C \cdot D$ (see Section 1.4). This concludes the proof.

Proof of Lemma 8.4. Suppose $v=a \log \sum_{i}\left|f_{i}\right|^{2}, v^{\prime}=a^{\prime} \log \sum_{i}\left|f_{i}^{\prime}\right|^{2}$, for holomorphic germs $f_{i}, f_{i}^{\prime} \in R$, and $a, a^{\prime}>0$. Let $\pi$ be a resolution of singularities of the curve $\left\{\prod_{i} f_{i} f_{i}^{\prime}=0\right\}$, i.e. its total transform has normal crossings. It suffices to prove that $\pi^{*}\left(v-v^{\prime}\right)$ is locally bounded at any point on $\pi^{-1}\{0\}$.

Pick a point $p \in \pi^{-1}(0)$. First suppose $p$ lies at the intersection of two irreducible components $E$ and $F$ of $\pi^{-1}\{0\}$. Choose coordinates such that $E \cup F$ is equal to $\{z=0\} \cup\{w=0\}$. As the curve $\left\{\prod_{i} \pi^{*} f_{i}=0\right\}$ has normal crossings, it is equal to $E \cup F$ locally. This means that for any $i$ we can write $\pi^{*} f_{i}=z^{c} w^{d} \xi$ for some $c, d \geq 0$ and a unit $\xi \in R$. We infer that the function $\pi^{*} v$ differs from $\operatorname{div}_{E}\left(\pi^{*} v\right) \log |z|+\operatorname{div}_{F}\left(\pi^{*} v\right) \log |w|$ by a bounded function. The same holds for $v^{\prime}$. As the tree transforms of $v$ and $v^{\prime}$ coincide, the values of the divisorial valuations $\nu_{E}$ and $\nu_{F}$ on $v$ and $v^{\prime}$ are equal. Hence $\pi^{*}\left(v-v^{\prime}\right)$ is bounded at $p$.

A similar argument applies when $p$ is a smooth point of $\pi^{-1}(0)$.

8.2. Lower bound. Our aim is to prove that one inequality in (8.1) holds without any restriction on the psh functions.

Proposition 8.5. Suppose $u, v$ are psh functions such that the wedge product $d d^{c} u \wedge d d^{c} v$ is admissible. Then

$$
d d^{c} u \wedge d d^{c} v\{0\} \geq \rho_{u} \cdot \rho_{v}=\iint_{\mathcal{V} \times \mathcal{V}} \mu \cdot \nu d \rho_{u}(\mu) d \rho_{v}(\nu),
$$

where $\rho_{u}, \rho_{v}$ are the tree measures of $u$ and $v$, respectively.

Note that in particular, when the right hand side in (8.2) is infinite, the wedge product $d d^{c} u \wedge d d^{c} v$ cannot be admissible.

In view of (5.2) and Theorem 6.1 we deduce the following and significantly weaker classical result (see [De1, Chapter III, Corollary 3.7.9]).

Corollary 8.6. If the wedge product $d d^{c} u \wedge d d^{c} v$ is admissible, then its mass at the origin is bounded from below by the product of the Lelong numbers of $u$ and $v$ at the origin. 
Proof of Proposition 8.5. Let $u_{n}, v_{n}$ be the Demailly approximants of $u$ and $v$, respectively (see Section 2.2). Using (2.7) and Proposition 8.2 we infer

$$
d d^{c} u \wedge d d^{c} v\{0\} \geq \limsup _{n \rightarrow \infty} d d^{c} u_{n} \wedge d d^{c} v_{n}\{0\}=\limsup _{n \rightarrow \infty} \rho_{n} \cdot \sigma_{n},
$$

where $\rho_{n}$ and $\sigma_{n}$ are the measures represented by $u_{n}$ and $v_{n}$, respectively. Now Proposition 5.17 gives $\lim \sup \rho_{n} \cdot \sigma_{n} \geq \rho \cdot \sigma$, completing the proof.

\subsection{The Hölder continuous case.}

Theorem 8.7. Suppose $\varphi$ is a psh weight for which $e^{\varphi}$ is Hölder continuous in a neighborhood of the origin. Then for any psh function $u$ we have

$$
d d^{c} u \wedge d d^{c} \varphi\{0\}=\rho_{u} \cdot \rho_{\varphi}=\iint_{\mathcal{V} \times \mathcal{V}} \mu \cdot \nu d \rho_{u}(\mu) d \rho_{\varphi}(\nu),
$$

where $\rho_{u}$ and $\rho_{\varphi}$ are the measures on $\mathcal{V}$ represented by $u$ and $\varphi$, respectively.

An equivalent formulation is

Corollary 8.8. Under the assumptions of Theorem 8.7, the generalized Lelong number defined by $\varphi$ is an average of valuations:

$$
\nu_{\varphi}(u)=\int_{\mathcal{V}} \mu(u) d \rho_{\varphi}(\mu)
$$

for any psh function $u$.

Corollary 8.9. Two psh weights whose exponentials are Hölder continuous define the same generalized Lelong numbers iff they have the same tree transform, or, equivalently, iff they have the same tree measure, or, yet equivalently, iff their pullbacks by any modification have the same Lelong numbers at any point on the exceptional divisor.

Remark 8.10. If $\varphi$ is a homogeneous psh weight in coordinates $(x, y)$, i.e. $\varphi(x, y)=$ $\Phi(|x|,|y|)=c^{-1} \Phi\left(|x|^{c},|y|^{c}\right)$, for all $c>0$, then its tree measure $\rho_{\varphi}$ is supported on the set of monomial valuations in $(x, y)$. In this case, Corollary 8.8 implies that $\nu_{\varphi}$ is an average of monomial valuations. This was proved by Rashkovskii [Ra, Corollary 1] in any dimension.

Proof of Theorem 8.7. Let $\varphi_{n}$ be the Demailly approximating sequence of $\varphi$ as in Section 2.2. As $e^{\varphi}$ is Hölder continuous, (2.2) gives

$$
\varphi-\frac{C}{n} \leq \varphi_{n} \leq\left(1-\frac{2}{n c}\right) \varphi+C,
$$

near the origin. Proposition 2.3 then implies $\nu_{\varphi}(u) \geq \nu_{\varphi_{n}}(u) \geq(1-2 / n c) \nu_{\varphi}(u)$, hence $d d^{c} u \wedge d d^{c} \varphi_{n}\{0\} \rightarrow d d^{c} u \wedge d d^{c} \varphi\{0\}$.

On the other hand (8.4) also gives $g_{\varphi_{n}} \geq g_{\varphi} \geq(1-2 / n c) g_{\varphi_{n}}$, where $g_{\varphi}$ and $g_{\varphi_{n}}$ are the tree transforms of $\varphi$ and $\varphi_{n}$, respectively. Thus

$$
\begin{aligned}
& d d^{c} u \wedge d d^{c} \varphi\{0\}=\lim _{n \rightarrow \infty} d d^{c} u \wedge d d^{c} \varphi_{n}\{0\}=\lim _{n \rightarrow \infty} \rho_{u} \cdot \rho_{\varphi_{n}}= \\
& =\lim _{n \rightarrow \infty} \int_{\mathcal{V}} g_{\varphi_{n}} d \rho_{u}=\int_{\mathcal{V}} g_{\varphi} d \rho_{u}=\rho_{u} \cdot \rho_{\varphi} .
\end{aligned}
$$


Here the second equality follows from Proposition 8.2 and the fourth from dominated convergence.

\section{REFERENCES}

[Bouc] S. Boucksom. Divisorial Zariski decompositions on compact complex manifolds. Ann. Sci. École Norm. Sup. (4) 37 (2004), no. 1, 45-76.

[BDPP] S. Boucksom, J.-P. Demailly, M. Paun, and T. Peternell. The pseudo-effective cone of a compact Kähler manifold and varieties of negative Kodaira dimension. www. arxiv.org/abs/math. AG/0405285.

[Bour] N. Bourbaki. Intégration. Hermann. Paris, 1965.

[De1] J.-P. Demailly. Complex analytic and algebraic geometry. Book available at the website www-fourier.ujf-grenoble.fr/ demailly/books.html.

[De2] J.-P. Demailly. Nombres de Lelong généralisés, théorèmes d'intégralité et d'analyticité. Acta Math. 159 (1987), no. 3-4, 153-169.

[De3] J.-P. Demailly. Regularization of closed positive currents and intersection theory. J. Algebraic Geom. 1 (1992), no. 3, 361-409.

[DK] J.-P. Demailly and J. Kollàr. Semi-continuity of complex singularity exponents and Kähler-Einstein metrics on Fano orbifolds. Ann. Sci. École Norm. Sup. 34 (2001), no. 4, 525-556.

[Du] R. Dujardin. Dynamique d'applications non polynomiales et courants laminaires. Thèse de l'université Orsay-Paris-XI, 2002.

[FJ1] C. Favre and M. Jonsson. The valuative tree. Lecture Notes in Mathematics, 1853. Springer-Verlag, Berlin, 2004.

[FJ2] C. Favre and M. Jonsson. Valuations and multiplier ideals. Available at www. arxiv.org/abs/math. CV/0406109.

[FJ3] C. Favre and M. Jonsson. Brolin's theorem for curves in two complex dimensions. Ann. Inst. Fourier. 53 (2003), no 5, 1461-1501.

[FJ4] C. Favre and M. Jonsson. Eigenvaluations. www.arxiv.org/abs/math.DS/0410417.

[G] V. Guedj. Desingularization of quasiplurisubharmonic functions. To appear in Internat. J. Math.

[Hö] L. Hörmander. Notions of convexity. Progress in Mathematics, 127. Birkhäuser. 1994.

[HP] J. H. Hubbard and P. Papadopol. Newton's method applied to two quadratic equations in $\mathbf{C}^{2}$ viewed as a global dynamical system. Preprint, 2001. Available at www .math.sunysb.edu/cgi-bin/preprint.pl?ims00-01.

[Ki1] C.-O. Kiselman. Un nombre de Lelong raffiné. Séminaire d'analyse complexe et géométrie 1985-1987, pp. 61-70. Faculté des sciences de Tunis et Faculté des sciences et techniques de Monastir.

[Ki2] C.-O. Kiselman. Attenuating the singularities of plurisubharmonic functions. Ann. Polon. Math. 60 (1994), no. 2, 173-197.

[Kw] J Kiwi. Puiseux series polynomial dynamics and iteration of complex cubic polynomials. Available at www.arxiv.org/abs/math.DS/0409397.

[Le] P. Lelong. Intégration sur un ensemble analytique complexe. Bull. Soc. Math. France 85 (1957), 239-262.

[Mi] S. K. Mimouni. Singularités des fonctions plurisousharmoniques et courants de Liouville. Thèse de la faculté des sciences de Monastir, Tunisie, 2001.

[Ra] A. Rashkovskii. Lelong numbers with respect to regular plurisubharmonic weights. Results Math. 39 (2001), no. 3-4, 320-332.

[Sp] M. Spivakovsky. Valuations in function fields of surfaces. Amer. J. Math. 112 (1990), no. 1, 107-156.

[St] Z. Słodkowski. Uniqueness property for positive closed currents in $\mathbf{C}^{2}$. Indiana Univ. Math. J. 48 (1999), no. 2, 635-652.

[Za] O. Zariski. The compactness of the Riemann manifold of an abstract field of algebraic functions. Bull. Amer. Math. Soc. 50 (1944). 683-691. 
CNRS-Université Paris 7, Institut de Mathématiques, Equipe Géométrie et Dynamique, F-75251 Paris Cedex 05, France

E-mail address: favre@math.jussieu.fr

Department of Mathematics, University of Michigan, Ann Arbor, Mi 48109-1109, USA

E-mail address: mattiasj@umich.edu

Department of Mathematics, Royal Institute of Technology, SE-100 44 StockHOLM, SWEDEN

E-mail address: mattiasj@kth.se 
